\title{
Interfaces, wetting, and capillary nematization of a hard-rod fluid: Theory for the Zwanzig model
}

\author{
René van Roij \\ Institute for Theoretical Physics, Utrecht University, Princetonpln 5, 3584 CC Utrecht, The Netherlands \\ Marjolein Dijkstra \\ Debye Institute, Condensed Matter Physics, Utrecht University, Princetonpln 5, 3584 CC Utrecht, \\ The Netherlands \\ Robert Evans \\ H. H. Wills Physics Laboratory, University of Bristol, Tyndall Avenue, Bristol BS8 1TL, United Kingdom
}

(Received 28 March 2000; accepted 21 June 2000)

\begin{abstract}
We investigate interfacial and capillary phenomena in a simple model for a fluid of hard rods, viz. the Zwanzig model, in which the orientations of rectangular blocks are restricted to three orthogonal directions. The theory, which is based on an Onsager-like free energy functional, predicts local biaxial ordering at the "free" interface between the coexisting isotropic and nematic phases. For an isotropic bulk fluid in contact with a single planar hard wall, we find a continuous surface phase transition from uniaxial to biaxial local symmetry, followed by complete wetting of the wallisotropic fluid interface by a nematic film with director parallel to the wall, as the reservoir density approaches its value at bulk coexistence. For a fluid confined by two parallel hard walls we determine a first-order capillary nematization transition at large wall separation, which terminates in a capillary critical point when the wall separation is about twice the length of the rods. This transition is the analog of the capillary condensation observed for simple fluids confined by attractive walls but is purely entropy driven here. (c) 2000 American Institute of Physics.

[S0021-9606(00)51535-9]
\end{abstract}

\section{INTRODUCTION}

Fluids of rodlike particles have attracted much attention over the years. Experimental observations date back to the 1920s and 1930s, when Zocher ${ }^{1}$ and Bawden et al. ${ }^{2}$ investigated colloidal systems of vanadiumpentoxide $\left(\mathrm{V}_{2} \mathrm{O}_{5}\right)$ and tobacco mosaic virus (TMV) particles, respectively, and found a phase transition from an isotropic fluid phase $(I)$ at low concentrations to an orientationally ordered nematic phase $(N)$ at higher concentrations. This density-driven isotropic nematic (IN) phase transition in a homogeneous bulk fluid of rodlike particles was first tackled theoretically in the 1940s, when Onsager argued that these colloidal rods can be modeled, under certains conditions, as mutually impenetrable hard rods. He accounted for the IN transition in terms of a competition between the maximization of orientational entropy and minimization of excluded volume. ${ }^{3}$ A key ingredient of Onsager's theory is the one-particle distribution as a function of the rod orientation, for which he derived a nonlinear integral equation. This equation is exact when the length-to-diameter ratio of the rods tends to infinity. At low bulk densities the only solution to this equation is a uniform constant, which describes the isotropic phase, while at sufficiently high densities nonuniform solutions of uniaxial symmetry exist, which describe the nematic phase. Although explicit calculation of these peaked nematic distributions is analytically intractable, this is numerically straightforward because the uniaxial symmetry reduces the problem to a onedimensional one in the polar angle of the rod orientation. The IN bulk coexistence that follows from Onsager's hard-rod theory is well established, with relative numerical uncertainties of about $1: 10^{4}$ for the coexisting densities and order parameters. ${ }^{4}$ Computer simulations in the 1980s showed that hard-rod systems can also form the liquid crystalline smectic- $A$ phase. ${ }^{5}$ The bulk phase diagram of hard spherocylinders has been determined as a function of density and length-to-diameter ratio by density functional theory ${ }^{6-8}$ and by simulation. ${ }^{9,10}$ Apart from some details, the general agreement between theory and simulation is good. Noting the experimental observation of the smectic- $A$ phase in a highdensity TMV suspension, ${ }^{11}$ one can conclude that many aspects of the bulk behavior of pure hard-rod fluids are rather well understood by now.

More complex and much richer phenomena occur in inhomogeneous hard-rod fluids. These include IN interfaces, surface effects arising from adsorption at an external wall, or capillary effects arising from spatial confinement. The main complication is caused by the simultaneous presence of anisotropy and inhomogeneity in the one-particle distribution. In the case of interest here, with a spatial inhomogeneity in the $z$ direction only, generally the one-particle distribution has a nontrivial dependence on the center-of-mass coordinate $z$, the polar angle $\theta$, and the azimuthal angle $\varphi$ of the $\operatorname{rod}$ orientation. In other words, the loss of translational invariance in the $z$ direction may also break the uniaxial symmetry about the nematic director, and the resulting symmetry of the equilibrium distribution may be biaxial. Taking into account the simultaneous presence of inhomogeneity and biaxiality is computationally demanding, and workers often resort to ap- 
proximations that simplify or discard either one or both of these features. For instance, Holyst and Poniewierski assume the "free" IN interface of hard spherocylinders, of length $L$ and diameter $D$, to be sharp, i.e., a step function at $z=0$, such that the one-particle distribution is that of the coexisting bulk isotropic phase for $z<0$ and that of the bulk nematic phase for $z>0$. Employing this approximation within an inhomogeneous generalization of Onsager's theory for bulk hard-rod fluids, they show that the IN interfacial tension is a minimum for a tilt angle of about $60^{\circ}$, independent of $L / D .^{12}$ The tilt angle is the angle between the surface normal and the bulk nematic director. This result is, however, in disagreement with more recent and extensive calculations by Moore and McMullen, ${ }^{13}$ Chen and Noorlandi, ${ }^{14}$ and Chen, ${ }^{15}$ who employ the same generalized Onsager functional but consider a wider class of variational profiles (smooth, finite width) for the IN interface. The resulting surface tensions are up to $50 \%$ lower than those of Ref. 12, and the most favorable tilt angle for long rods is found to be $90^{\circ}$, i.e., parallel to the surface. ${ }^{13-15}$ In another study, Poniewierski and Holyst use the generalized Onsager functional to describe a fluid of hard rods in contact with a planar hard wall located at $z$ $=0 .{ }^{16}$ Here an approximation is made that gives rise to bulk distributions for $z>(L+D) / 2$. The surface tensions that follow from these approximate profiles, combined with those of the IN interface obtained in Ref. 12, imply that a nematic film with a director parallel to the surface wets completely the hard wall-isotropic fluid interface as the bulk density approaches that of the IN transition. ${ }^{16}$ Interestingly, this conclusion points to an internal inconsistency since the approximation for the one-particle distribution does not allow for the diverging thickness of a wetting film. Moreover, the approximation does not allow for the possibility of a biaxial profile, which is a prerequisite for the description of a nematic film with a director parallel to the wall. The issue of biaxiality is addressed in a more recent paper by Poniewierski, where the stability of uniaxial profiles close to the wall is studied by means of a bifurcation analysis. ${ }^{17}$ The onset of biaxial symmetry occurs for profiles corresponding to a bulk density $15 \%$ below that of the coexisting isotropic bulk fluid, demonstrating the relevance of surface biaxiality even for states that are deep in the isotropic bulk phase. The nature of the uniaxial-biaxial transition (first order or continuous), or that of the wetting films, was not addressed in Ref. 17-this requires a (computationally costly) higher-order bifurcation analysis and full numerical calculations of the biaxial profiles. We are aware of only one study, by Chen and Cui, that does describe full calculations of density inhomogeneities coupled to biaxiality. ${ }^{18}$ These authors study a fluid of hardcore semiflexible polymers near a hard wall, using a density functional that resembles the inhomogeneous Onsager functional. They find a very weakly first-order uniaxial-biaxial transition at a chemical potential substantially below the IN bulk transition. They also find a (biaxially symmetric) nematic film whose thickness diverges as bulk IN coexistence is approached. ${ }^{18}$ This finding lends very strong support to the conjecture that the hard wall-isotropic fluid interface is wet completely by a nematic phase with a director parallel to the wall. However, no attempt was made to confirm the conjec- ture via surface tension calculations. Complete wetting requires the wall-isotropic fluid tension to be the sum of the wall-nematic fluid tension and the IN tension. Testing this equality requires accurate calculation of all the three surface tensions, which can be obtained but only by even larger computational efforts.

Even less work has been devoted to systems of confined hard-rod fluids. Here we mention very recent work by Allen on hard ellipsoids in external anchoring fields, ${ }^{19,20}$ which describes theoretically determined biaxial profiles and simulation results as well. The focus of Ref. 19 is on elasticity and anchoring of liquid crystals, and that of Ref. 20 on the structure of the IN interface. The work of Refs. 19 and 20 is not, as is the present paper, concerned with phase transitions of the confined fluid. Another recent study by Mao et al. is concerned with hard spherocylinders confined by two parallel hard plates at separation $H .{ }^{21}$ The focus of Ref. 21 is on the $H$ dependence of the excess free energy, and on the solvation force exerted by the rod fluid on the plates. Their aim is to understand depletion interactions in colloidal systems. Attention is restricted to the uniaxially symmetric lowdensity regime. By analogy with the single-wall results discussed above, one expects, as the authors of Ref. 21 also point out, a uniaxial-biaxial surface transition to occur at both walls for high reservoir densities. Moreover, by analogy with the behavior of confined simple fluids ${ }^{22,23}$ and thermotropic liquid crystals, ${ }^{24,25}$ it is tempting to speculate that the analog of capillary condensation, i.e., capillary nematization, should occur in the confined hard-rod system. Such a transition corresponds to a shift of the bulk IN transition to a lower chemical potential or reservoir density, and should occur provided the walls prefer the nematic phase. The spherical symmetry of the pair interactions in simple liquids, and the assumed incompressibility of thermotropic liquid crystals (resulting in homogeneous density profiles), permit detailed studies of interfaces between coexisting phases, wetting against substrates, capillary phenomena, and the interplay between them. Most of these investigations are within the framework of either density functional theory or Landau-de Gennes theory. It is our impression that quantitative studies of the corresponding phenomena in density-driven hard-core liquid crystals (or lyotropic liquid crystals) have been hindered by the numerical difficulties in determining inhomogeneous biaxially symmetric equilibrium profiles. It is against this background that we undertake the present study.

In this paper we consider a fluid of hard rods of rectangular shape with orientations restricted to three (mutually perpendicular) directions. The bulk IN transition of this model was considered by Zwanzig in $1963,{ }^{26}$ while the nematic-smectic bulk transition was studied only recently. ${ }^{27}$ Although a comparison of the results of the so-called Zwanzig model with those of models with continuous orientations is not entirely straightforward, ${ }^{28}$ these studies do show that the Zwanzig model captures the essential symmetries and mechanisms that underly the bulk phase transitions of more sophisticated models of bulk hard-rod fluids. Moreover, its simplicity facilitates the (numerical) analysis considerably. Here we show that the determination of inhomogeneous biaxial profiles is numerically straightforward, at least within 
Onsager's second virial free energy functional for the Zwanzig model. This allows us to make a quantitative and consistent study of several aspects of inhomogeneous hard-rod fluids. We focus, within a single theory, on the IN interface, the uniaxial-biaxial surface transition and wetting against a single hard wall, and capillary nematization for the fluid between two parallel hard walls. A brief report describing some of these results has been published elsewhere. ${ }^{29}$

\section{DENSITY FUNCTIONAL FOR THE ZWANZIG MODEL}

We consider a system of rectangular hard rods of size $L \times D \times D$ (with $L \gg D$ ) in a three dimensional space spanned by the orthonormal unit vectors $\hat{x}_{\alpha}$ with $\alpha=1,2,3$ representing $\hat{x}, \hat{y}$, and $\hat{z}$, respectively. The position of the center of mass $\mathbf{r}$ of a rod is continuous, while the allowed orientations of the three principal axes of each rod is restricted to directions $\hat{x}_{\alpha}$. Due to up-down symmetry and the degeneracy of the two short axes of a rod, its orientation can be represented by the discrete variable $\alpha=1,2,3$, corresponding to the orientation of the long axis of the rod along $\hat{x}_{\alpha}$. We are concerned with the equilibrium one-particle distribution $\rho(\mathbf{r}, \alpha)$ $\equiv \rho_{\alpha}(\mathbf{r})$ and the phase behavior of this system of rods in a given external potential $V(\mathbf{r}, \alpha) \equiv V_{\alpha}(\mathbf{r})$ at a fixed chemical potential $\mu$, the total volume of the system being $V$. To this end, we consider the grand-potential functional

$$
\Omega[\rho]=\mathcal{F}[\rho]+\sum_{\alpha=1}^{3} \int d \mathbf{r} \rho_{\alpha}(\mathbf{r})\left(V_{\alpha}(\mathbf{r})-\mu\right),
$$

which is minimized by the equilibrium one-particle distribution, the minimal value being the equilibrium grand potential of the system. ${ }^{30}$ Here $\mathcal{F}[\rho]$ is the intrinsic free energy functional, ${ }^{30}$ which we assume to be given by

$$
\begin{aligned}
\beta \mathcal{H}[\rho]= & \sum_{\alpha=1}^{3} \int d \mathbf{r} \rho_{\alpha}(\mathbf{r})\left(\ln \left(\rho_{\alpha}(\mathbf{r}) \Lambda^{3}\right)-1\right) \\
& -\frac{1}{2} \sum_{\alpha, \alpha^{\prime}} \int d \mathbf{r} d \mathbf{r}^{\prime} f_{\alpha \alpha^{\prime}}\left(\mathbf{r}, \mathbf{r}^{\prime}\right) \rho_{\alpha}(\mathbf{r}) \rho_{\alpha^{\prime}}\left(\mathbf{r}^{\prime}\right),
\end{aligned}
$$

where $\beta=1 / k_{B} T$, with $T$ the temperature and $\Lambda$ the thermal wavelength. $f_{\alpha \alpha^{\prime}}\left(\mathbf{r}, \mathbf{r}^{\prime}\right)$ is the Mayer function, which for hard-core interactions equals -1 if the particles overlap and is 0 otherwise. The functional Eq. (2) is the discrete and inhomogeneous version of Onsager's famous second virial functional used, e.g., in Refs. 12, 16, 17, 21, and 31 to describe bulk, surface and confining phenomena for fluids of freely rotating hard rods. By considering $\rho_{\alpha}(\mathbf{r})=\rho(z) \delta_{\alpha 3}$, with $\delta_{i j}$ the Kronecker delta, Eq. (2) also reduces to the second virial functional employed by Mulder in his studies of the bulk nematic-smectic transition of perfectly aligned hard rods. ${ }^{32}$ Moreover, this functional is identical to one used by Ratón et al. to study the influence of the coupling of orientational and positional ordering on the nematic-smectic transition. $^{27}$ In this paper we do not consider crystalline, smectic, columnar, or other spatially ordered bulk phases of hard-rod systems. ${ }^{33} \mathrm{We}$ restrict our attention to external potentials of the type $V_{\alpha}(\mathbf{r})=V_{\alpha}(z)$. Thus, we only consider profiles $\rho_{\alpha}(\mathbf{r})=\rho_{\alpha}(z)$. The macroscopic interfacial area in the $x y$ plane is denoted by $A$.
Defining $S_{i \alpha}=D+(L-D) \delta_{i \alpha}$, which represents the spatial extent in direction $i=1,2,3$ of a rod with orientation $\alpha$, we can can write the Mayer function of the rods explicitly as

$$
f_{\alpha \alpha^{\prime}}\left(\mathbf{r}, \mathbf{r}^{\prime}\right)=-\prod_{i=1}^{3} \Theta\left(\frac{1}{2}\left(S_{i \alpha}+S_{i \alpha^{\prime}}\right)-\left|r_{i}-r_{i}^{\prime}\right|\right),
$$

where $r_{i}=\mathbf{r} \cdot \hat{x}_{i}$ and $\Theta(t)$ is the Heaviside step function. This particular factorization of the Mayer function, which results from the combination of particle shape and restricted orientations, is the key to the relative simplicity of the calculations that follow, compared to those of say spherocylinders with continuous orientations. Introducing the dimensionless densities $c_{\alpha}(z)=L^{2} D \rho_{\alpha}(z)$, we can rewrite Eqs. (2) with (3) as

$$
\begin{aligned}
\frac{\beta \mathcal{F}[c] L^{2} D}{A}= & \sum_{\alpha} \int d z c_{\alpha}(z)\left(\ln c_{\alpha}(z)-1\right) \\
& +\frac{1}{2} \sum_{\alpha, \alpha^{\prime}} \int d z d z^{\prime} K_{\alpha \alpha^{\prime}}\left(z, z^{\prime}\right) c_{\alpha}(z) c_{\alpha^{\prime}}\left(z^{\prime}\right),
\end{aligned}
$$

where we set $\Lambda^{3}=L^{2} D$ without loss of generality. The kernel $K$ is symmetric, $K_{i j}=K_{j i}$, and satisfies $K_{22}=K_{11}$ and $K_{23}=K_{13}$ by symmetry, with

$$
\begin{aligned}
& K_{11}\left(z, z^{\prime}\right)=\frac{E_{\|}}{L^{2} D} \frac{\Theta\left(D-\left|z-z^{\prime}\right|\right)}{2 D}, \\
& K_{33}\left(z, z^{\prime}\right)=\frac{E_{\|}}{L^{2} D} \frac{\Theta\left(L-\left|z-z^{\prime}\right|\right)}{2 L}, \\
& K_{12}\left(z, z^{\prime}\right)=\frac{E_{\perp}}{L^{2} D} \frac{\Theta\left(D-\left|z-z^{\prime}\right|\right)}{2 D}, \\
& K_{13}\left(z, z^{\prime}\right)=\frac{E_{\perp}}{L^{2} D} \frac{\Theta\left(\frac{1}{2}(L+D)-\left|z-z^{\prime}\right|\right)}{L+D},
\end{aligned}
$$

with $E_{\|}=8 L D^{2}$ and $E_{\perp}=2 D(L+D)^{2}$ the excluded volume of a pair of parallel and perpendicular rods, respectively. In the limit $D / L \rightarrow 0$, which we adopt from now on, the diagonal elements $K_{\alpha \alpha}\left(z, z^{\prime}\right)$ contribute terms that are smaller than the off-diagonal elements by a factor of order $D / L$. Thus, the $K_{\alpha \alpha}$ contributions in Eq. (4) will be neglected. Strictly speaking this approximation is only justified if $c_{\alpha}(z)$ is of the same order for each $\alpha$, i.e., in the isotropic phase and in the nematic phase not too far above the isotropicnematic transition density; it is no longer justified in the perfectly aligned nematic phase. Recognizing that $\lim _{D \rightarrow 0} \Theta\left(D-\left|z-z^{\prime}\right|\right) /(2 D)=\delta\left(\left|z-z^{\prime}\right|\right)$, the Dirac delta, and that $\lim _{D / L \rightarrow 0} E_{\perp} / L^{2} D=2$, the grand potential functional is from Eqs. (1), (4), and (5), given by

$$
\begin{aligned}
\frac{\beta \Omega[c] L^{2} D}{A}= & \sum_{\alpha=1}^{3} \int d z c_{\alpha}(z)\left(\ln c_{\alpha}(z)-1-\beta \mu\right. \\
& \left.+\beta V_{\alpha}(z)\right)+2 \int d z\left(c_{1}(z) c_{2}(z)\right. \\
& \left.+\left(c_{1}(z)+c_{2}(z)\right) \bar{c}_{3}(z)\right),
\end{aligned}
$$

where we introduced 


$$
\begin{aligned}
\bar{c}_{\alpha}(z) & =\frac{1}{L} \int d z^{\prime} \Theta\left(\frac{1}{2} L-\left|z-z^{\prime}\right|\right) c_{\alpha}\left(z^{\prime}\right) \\
& =\frac{1}{L} \int_{z-L / 2}^{z+L / 2} d z^{\prime} c_{\alpha}\left(z^{\prime}\right) .
\end{aligned}
$$

The scaling of the dimensionless density $c$ is such that the right-hand side of Eq. (6) is independent of $D$ in the limit $D / L \rightarrow 0$, so that the only relevant length is $L$. The EulerLagrange equations resulting from the stationarity conditions $\delta \Omega[c] / \delta c_{\alpha}(z)=0$ can be cast in the form

$$
\begin{aligned}
& \ln c_{1}(z)=\beta \mu-\beta V_{1}(z)-2 c_{2}(z)-2 \bar{c}_{3}(z), \\
& \ln c_{2}(z)=\beta \mu-\beta V_{2}(z)-2 c_{1}(z)-2 \bar{c}_{3}(z), \\
& \ln c_{3}(z)=\beta \mu-\beta V_{3}(z)-2 \bar{c}_{1}(z)-2 \bar{c}_{2}(z) .
\end{aligned}
$$

Below we solve this set of three coupled nonlinear equations iteratively for fixed external potentials $V_{\alpha}(z)$ and chemical potentials $\mu$. In all numerical calculations we use an equidistant $z$ grid of at least 100 points per $L$, with some checks using 200 or 500 points per $L$ which give virtually identical results. Convergence is assumed when the relative difference between the results of iteration $j$ and $j+1$ is smaller than $10^{-7}$ for all values of $z$ in the grid; this proves sufficient for the required accuracy.

The interpretation of the numerical results and the derivation of some analytic results is facilitated by considering three independent combinations of $c_{\alpha}(z)$, viz.

$$
\begin{aligned}
& c(z)=c_{1}(z)+c_{2}(z)+c_{3}(z), \\
& s(z)=\frac{c_{3}(z)-\frac{1}{2}\left(c_{1}(z)+c_{2}(z)\right)}{c(z)}, \\
& \Delta(z)=\frac{c_{1}(z)-c_{2}(z)}{c(z)},
\end{aligned}
$$

or reversibly

$$
\begin{aligned}
& c_{1}(z)=\frac{1}{3} c(z)\left(1-s(z)+\frac{3}{2} \Delta(z)\right), \\
& c_{2}(z)=\frac{1}{3} c(z)\left(1-s(z)-\frac{3}{2} \Delta(z)\right), \\
& c_{3}(z)=\frac{1}{3} c(z)(1+2 s(z)) .
\end{aligned}
$$

Clearly, $c(z)$ represents the total density profile, while $s(z)$ and $\Delta(z)$ are orientational order parameter profiles. This choice is such that: (i) $s(z)=\Delta(z)=0$ corresponds to a (locally) isotropic distribution; (ii) $-\frac{1}{2} \leqslant s(z) \leqslant 1$ with $\Delta(z)$ $=0$ is a uniaxial distribution with symmetry axis $\hat{z}$, where a positive/negative sign of $s(z)$ signals preference/depletion of particles oriented parallel to the symmetry axis; (iii) 0 $\neq|\Delta(z)| \neq|2 s(z)|$ describes a distribution of biaxial symmetry, where a positive or negative sign of $\Delta(z)$ signals preference for orientations parallel to $\hat{x}$ or $\hat{y}$, respectively. If, however, $\Delta(z)=\mp 2 s(z) \neq 0$, then the system is still (locally) uniaxial, but with a symmetry axis $\hat{x}$ (minus: $c_{2}(z)=c_{3}(z)$ ) or $\hat{y}$ (plus: $\left.c_{1}(z)=c_{3}(z)\right)$ instead of $\hat{z}$. In this case, it is convenient to introduce the profiles $s^{\prime}(z)=-\frac{1}{2} s(z) \pm \frac{3}{4} \Delta(z)$ and $\Delta^{\prime}(z)=s(z) \pm \frac{1}{2} \Delta(z)$, which then play the role of uniaxial and biaxial order parameters, respectively. The defi- nitions [Eqs. (9) and (10)] are such that the three independent linear combinations of the Euler-Lagrange equations (8) read

$$
\begin{aligned}
\begin{aligned}
\ln \frac{c_{1}(z)}{c_{2}(z)}=-\beta( & \left.V_{1}(z)-V_{2}(z)\right)+2 c(z) \Delta(z) \\
\ln \frac{c_{1}(z) c_{2}(z)}{c_{3}^{2}(z)}= & -\beta\left(V_{1}(z)+V_{2}(z)-2 V_{3}(z)\right) \\
& -\frac{4}{3} c(z)(1-s(z))-\frac{4}{3 L} \int_{z-L / 2}^{z+L / 2} d z^{\prime} c\left(z^{\prime}\right) \\
& \times\left(-1+4 s\left(z^{\prime}\right)\right), \\
\ln c_{3}(z)=\beta \mu- & \beta V_{3}(z) \\
- & \frac{4}{3 L} \int_{z-L / 2}^{z+L / 2} d z^{\prime} c\left(z^{\prime}\right)\left(1-s\left(z^{\prime}\right)\right)
\end{aligned}
\end{aligned}
$$

which, for fixed $\beta V_{\alpha}(z)$ and $\beta \mu$, form a closed set of equations for $c(z), s(z)$, and $\Delta(z)$ if one substitutes Eq. (10) into the left hand-sides of Eq. (11).

\section{ISOTROPIC AND NEMATIC BULK PHASES}

We consider first the homogeneous bulk fluid with $V_{\alpha}(\mathbf{r})=0$ in the macroscopic volume of interest. The equilibrium profiles are then constant: $c(z)=c_{b}, s(z)=s_{b}$, and $\Delta(z)=0$, where $c_{b}$ and $s_{b}$ are the bulk values of the total density and the nematic order parameter, respectively, determined by $\beta \mu$, and where the biaxiality vanishes because of symmetry. Inserting these constant profiles into the EulerLagrange equations (11) reveals that the first equation is satisfied identically, while the second one can be rewritten as

$$
\ln \left(\frac{1+2 s_{b}}{1-s_{b}}\right)=2 c_{b} s_{b} .
$$

It is easily determined that the isotropic distribution, with $s_{b}=0$, is a solution of Eq. (12) at any $c_{b}$, and that additional nematic distributions, with $0<s_{b}<1$, are possible at sufficiently high $c_{b}$. The relation between nonzero $s_{b}$ and $c_{b}$ can be determined numerically from Eq. (12). A simple estimate of the minimum density required to obtain possible nonzero values of $s_{b}$ involves the low- $s_{b}$ expansion: $\ln \left[\left(1+2 s_{b}\right) /(1\right.$ $\left.\left.-s_{b}\right)\right] \simeq 3 s_{b}$, which equals the right-hand side of Eq. (12) at the so-called bifurcation density $c_{b}=c_{\mathrm{IN}}^{*}=3 / 2$. We stress, however, that nonzero values for $s_{b}$ are also possible for $c_{b}$ not too far below $c_{\mathrm{IN}}^{*}$.

In order to calculate the bulk phase coexistence of the $I$ and $N$ phase, we consider the chemical potential $\mu$ and the pressure $p=-\Omega_{0} / V$, where $\Omega_{0}$ is the minimal value of the functional Eq. (6). Inserting the constant profiles under consideration into Eq. (6) and the third one of Eq. (11) yields, after some algebra involving Eqs. (10),

$$
\begin{aligned}
& \beta p L^{2} D=c_{b}+\frac{2}{3} c_{b}^{2}\left(1-s_{b}^{2}\right), \\
& \beta \mu=\ln c_{b}+\ln \frac{1+2 s_{b}}{3}+\frac{4}{3} c_{b}\left(1-s_{b}\right) .
\end{aligned}
$$


Phase coexistence occurs between the isotropic state at $c_{b}$ $=c_{I}$ (with $\left.s_{b}=0\right)$ and the nematic state at $c_{b}=c_{N}$ [with $s_{b}$ $=s_{N}>0$ determined numerically from Eq. (12)] for which $\mu\left(c_{I}\right)=\mu\left(c_{N}\right)$ and $p\left(c_{I}\right)=p\left(c_{N}\right)$. A straightforward numerical calculation yields

$$
\begin{aligned}
& c_{I}=1.25822486, \\
& c_{N}=1.91544377, \\
& s_{N}=0.91498627,
\end{aligned}
$$

from which the values of the chemical potential $\mu_{\mathrm{IN}}$ and pressure $p_{\mathrm{IN}}$ at coexistence follow: $\beta \mu_{\mathrm{IN}}=0.8087227$ and $\beta p_{\text {IN }} L^{2} D=2.313645$. These coexistence data are identical to those reported by Zwanzig within the second virial approximation, ${ }^{26}$ apart from an (irrelevant) shift of $\ln 3$ in the definition of $\beta \mu$ and $\beta \mu_{\mathrm{IN}}$ in Ref. 26. For the latter reference we note that the numerical value of $p_{\text {IN }}$ is substantially smaller than the corresponding bulk coexistence pressure $p_{\text {IN }}^{\text {free }}$ of infinitely elongated, freely rotating hard spherocylinders (with continuous orientation degrees of freedom), i.e., $\beta p_{\text {IN }}^{\text {free }} L^{2} D=14.11 .^{4}$ Thus we have $p_{\text {IN }} / p_{\text {IN }}^{\text {free }} \simeq 0.16$. The reason for this substantial difference is that the orientational entropy of the Zwanzig model is much reduced due to the restricted number of allowed orientations, which causes (i) the IN transition to occur at a relatively low density, and (ii) the orientational order of the coexisting nematic phase to be relatively high. This was also pointed out by Straley. ${ }^{28}$ Note that the accuracy of the bulk coexistence data is higher than that obtained for rods with continuous orientation degrees of freedom. The difference arises from the fact that the stationarity condition Eq. (12) is an algebraic equation, whereas its continuous counterpart is a nonlinear integral equation. ${ }^{3}$ That we can obtain very accurate values for bulk coexistence data turns out to be relevant for the study of the interfacial properties and phase behavior to be discussed in subsequent sections.

\section{THE ISOTROPIC-NEMATIC INTERFACE}

We can create a planar interface between the coexisting isotropic and nematic bulk phase by imposing arbitrarily weak external potentials $V_{\alpha}(z)$, and in this mean-field treatment we can determine the structure of the "free" interface by calculating $c_{\alpha}(z)$ from Eqs. (8) with $\mu=\mu_{\mathrm{IN}}$ and $V_{\alpha}(z)$ $=0$, subject to appropriate boundary conditions. For a sufficiently large $z_{m}>0$, we impose the coexisting isotropic bulk phase at positions $z<-z_{m}$ by setting

$$
c_{\alpha}(z)=\frac{1}{3} c_{I} \quad\left(z<-z_{m}, \alpha=1,2,3\right),
$$

with $c_{I}$ given in Eq. (14). At $z>z_{m}$ we impose a nematic bulk phase at total density $c_{N}$. There are, however, two essentially different possibilities for the relative orientation of the director $\hat{n}$ of this nematic phase: perpendicular $(\perp)$ or parallel $(\|)$ to the interface, where we adopt the convention that $\hat{n}=\hat{x}$ (or equivalently $\hat{n}=\hat{y}$ ) is "parallel" to the interface, while $\hat{n}=\hat{z}$ is "perpendicular" to the interface. These two alternatives are represented by the boundary conditions

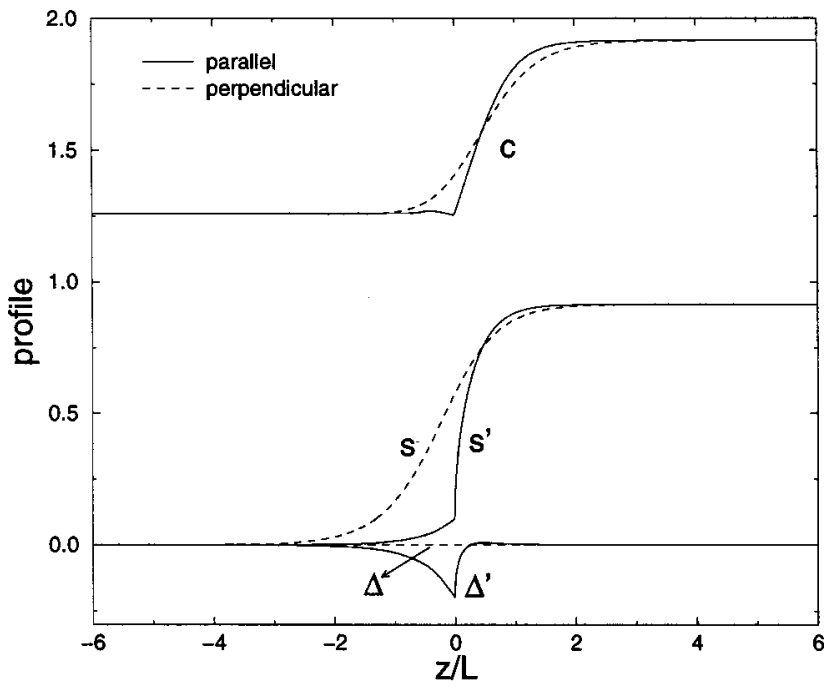

FIG. 1. Profiles characterizing the isotropic-nematic interface of the Zwanzig hard-rod fluid, as follows from solving the Euler-Lagrange equations (8) with $\mu=\mu_{\mathrm{IN}}$. The full and dashed curves denote perpendicular and parallel orientation, respectively, of the nematic director in bulk $(z \rightarrow+\infty)$ to the interfacial normal. $c$ represents the total density, $s$ is the uniaxial order parameter, and $\Delta \equiv 0$ the biaxial order parameter, for the perpendicular case. $s^{\prime}$ and $\Delta^{\prime}$ are the corresponding quantities for the parallel case.

$$
\begin{aligned}
\|: \quad c_{2,3}(z)= & \frac{1}{3} c_{N}\left(1-s_{N}\right), \quad c_{1}(z)=\frac{1}{3} c_{N}\left(1+2 s_{N}\right), \\
& \left(z>z_{m}\right) ; \\
\perp: \quad c_{1,2}(z)= & \frac{1}{3} c_{N}\left(1-s_{N}\right), \quad c_{3}(z)=\frac{1}{3} c_{N}\left(1+2 s_{N}\right), \\
& \left(z>z_{m}\right) .
\end{aligned}
$$

It is easily determined that the perpendicular geometry is of uniaxial symmetry, i.e., $s(z)=s_{N}$ and $\Delta(z)=0$ for $z>z_{m}$, while the parallel geometry is biaxial, i.e., $s(z)=-s_{N} / 2$ and $\Delta(z)=-2 s(z)=s_{N}$ for $z>z_{m}$. However, the nematic bulk phases far from the interface are related by a trivial rotation, and therefore it is convenient to compare the combinations $c(z), s(z)$, and $\Delta(z)$ of the uniaxial interface $(\perp)$ with $c(z)$, $s^{\prime}(z)$, and $\Delta^{\prime}(z)$ of the biaxial interface $(\|)$. These combinations, see below Eq. (10), are such that $s^{\prime}(z)=s_{N}$ and $\Delta^{\prime}(z)=0$, for $z>z_{m}$, i.e., the primed and unprimed profiles are identical far away from the interface. In Fig. 1 we plot these particular combinations of the profiles $c_{\alpha}(z)$ resulting from an iterative numerical solution of the Euler-Lagrange equations (8) satisfying the boundary conditions, Eqs. (15) and (16). The dashed and full curves represent the perpendicular and parallel geometry, respectively. Calculation of the profiles was performed for $z_{m} / L=10$ on an equidistant $z$ grid of 100 points per $L$; calculations made using 200 points per $L$ or $z_{m} / L=20$ gave indistinguishable results. Good convergence was only obtained by inputting very accurate bulk values for $c_{I}, c_{N}$, and $s_{N}$ in the asymptotic regime; poorer estimates caused the interface to "wander away" from $z$ $=0$, thereby preventing convergence. It is seen from Fig. 1 that the interfacial width is considerably larger for the perpendicular geometry, the profiles of which are relatively smooth and exhibit uniaxial character throughout since $\Delta(z) \equiv 0$. In contrast, the case of parallel relative orientation shows sharper features in all three profiles. There is a weak 
oscillation in the density $c(z)$ and a pronounced negative biaxiality $\Delta^{\prime}(z)$ on the isotropic side of the interface. The biaxiality becomes slightly positive on the nematic side before decaying quickly to zero. The negative sign of $\Delta^{\prime}(z)$ on the isotropic side signals a local depletion of rods "sticking through" the interface such that their end points enter the nematic side. This effect, and the equivalent sign change of the biaxiality, were obtained by Chen for hard rods with continuous orientations, ${ }^{15}$ although the amplitude of the biaxial order parameter calculated in Ref. 15 is smaller. Note that the order parameter profile $s^{\prime}(z)$ increases much more rapidly than the density profile $c(z)$ on the nematic side. This was also found by Chen. ${ }^{15}$

The calculated equilibrium profiles can be used to determine the interfacial tensions $\gamma_{\mathrm{IN}, \|}$ and $\gamma_{\mathrm{IN}, \perp}$ by inserting them back into the functional of Eq. (6) to yield the equilibrium value $\Omega_{0}$ of the grand potential of the system. Using the general definition of the surface tension as the surface excess grand potential per unit area,

$$
\gamma=\frac{\Omega_{0}+p V}{A},
$$

we obtain with the bulk pressure $p=p_{\text {IN }}$ the numerical values

$$
\begin{aligned}
& \beta \gamma_{\mathrm{IN}, \|} L D=(2.7960 \pm 0.0004) \times 10^{-2}, \\
& \beta \gamma_{\mathrm{IN}, \perp} L D=(5.0660 \pm 0.0002) \times 10^{-2} .
\end{aligned}
$$

The smaller value, by almost a factor 2 , is for the biaxial "parallel" configuration, which implies that a nematic director parallel to the IN interface is thermodynamically more favorable than a director perpendicular to the interface. This is consistent with calculations of the IN surface tension $\gamma_{\mathrm{IN}}^{\text {free }}\left(\theta_{t}\right)$ of freely rotating hard spherocylinders as a function of the (continuous) tilt angle $\theta_{t}$ between the surface normal and the nematic director as presented in Refs. 12-14. Although these studies reveal a nontrivial, and possibly nonmonotonic dependence on $\theta_{t}$, they all find that for the angles with an analog in the present model (parallel: $\theta_{t}=90^{\circ}$, perpendicular: $\left.\theta_{t}=0^{\circ}\right) \gamma_{\mathrm{IN}}^{\text {free }}\left(90^{\circ}\right)<\gamma_{\mathrm{IN}}^{\text {free }}\left(0^{\circ}\right) .^{12-14}$ It is also interesting to compare the numerical value of the lower tension $\gamma_{\mathrm{IN}} \equiv \gamma_{\mathrm{IN}, \|}$ given in Eq. (18) with the result $\gamma_{\mathrm{IN}}^{\text {free }}$ $\equiv \gamma_{\mathrm{IN}}^{\text {free }}\left(90^{\circ}\right)$ for infinitely elongated freely rotating spherocylinders. Without making any parametrization of profiles within the Onsager theory, Chen obtained $\beta \gamma_{\mathrm{IN}}^{\text {free }} L D=0.181$ $\pm 0.002 .{ }^{15}$ Thus our tension is substantially smaller than $\gamma_{\mathrm{IN}}^{\text {free }}$. Interestingly, however, the surface excess grand potential ratio $\gamma_{\mathrm{IN}} / \gamma_{\mathrm{IN}}^{\text {free }} \simeq 0.15$ is strikingly similar to the bulk grand potential ratio $p_{\text {IN }} / p_{\text {IN }}^{\text {free }} \simeq 0.16$ determined in Sec. III. This is another indication that while the Zwanzig model does indeed capture some of the essential physics of rod fluids, it renormalizes the numerical values of thermodynamic quantities compared to models with continuous orientations.

Finally we remark that the fractional accuracy of $\gamma_{\mathrm{IN}, \perp}$ is better than for $\gamma_{\mathrm{IN}, \|}$-see Eq. (18). This is due to the relatively smooth uniaxial profiles, which are slightly less sensitive to the discrete $z$ grid than the sharper biaxial profiles. This feature of the numerics is found throughout this work.

\section{THE FLUID IN CONTACT WITH A SINGLE HARD WALL}

In this section we consider a bulk fluid of rods (at chemical potential $\mu$ or bulk density $c_{b}$ ) in contact with a single wall. Here we restrict attention to a planar hard wall of (macroscopic) area $A$, surface normal $\hat{z}$, positioned at $z=0$. It is described by the external potential

$$
\beta V_{\alpha}^{(1)}(z)=\left\{\begin{array}{l}
\infty \quad \text { for } z<0 \quad(\alpha=1,2) \\
z<L / 2 \quad(\alpha=3), \\
0 \quad \text { otherwise }
\end{array}\right.
$$

where $z$ refers to the midpoint of a rod. The immediate consequence of such a wall is that rods oriented perpendicular to the wall, i.e., rods characterized by $\alpha=3$, cannot approach it closer than a distance $L / 2$, thereby giving rise to orientational order close to the wall for any bulk fluid in contact with it. At low $c_{b}$ one expects this wall-induced orientational order to preserve the symmetry between $\alpha=1,2$, i.e., $c_{1}(z)$ $=c_{2}(z)$ for $z<L / 2$. This symmetry will be refered to as "uniaxial" after its continuous analogue, the symmetry axis being the surface normal. In the liquid crystal literature this symmetry is often called "random planar." At sufficiently high $c_{b}$ one could expect that the tendency of rods to align might break this uniaxial surface symmetry, and then $c_{1}(z)$ $\neq c_{2}(z)$ for $z<L / 2$. The symmetry close to the wall is then referred to as "biaxial." The uniaxial-biaxial (UB) transition occurs for a value of $c_{b}$ well below the bulk transition density $c_{I}$. We can also enquire about the hard wallisotropic fluid interface in the limit $c_{b} \rightarrow c_{I}^{-}$, i.e., the wetting properties of the fluid of Zwanzig rods in contact with the hard wall. Both topics are investigated in some detail below.

In order to study the possible onset of biaxiality, we rewrite the first Euler-Lagrange equation (11) with $V_{\alpha}(z)$ $=V_{\alpha}^{(1)}(z)$ as

$$
\ln \frac{1-s(z)+\frac{3}{2} \Delta(z)}{1-s(z)-\frac{3}{2} \Delta(z)}=2 c(z) \Delta(z) .
$$

Equation (20) has a trivial uniaxial solution $\Delta(z)=0$, independent of $s(z)$ and $c(z)$. Nontrivial biaxial solutions $\Delta(z)$ $\neq 0$ are possible if $c(z) \geqslant c_{\mathrm{UB}}(z)$, where the UB bifurcation density $c_{\mathrm{UB}}(z)$ follows from a low- $\Delta$ expansion of the logarithm in Eq. (20) and is given by

$$
c_{\mathrm{UB}}(z)=\frac{3}{2(1-s(z))} .
$$

Note that $\Delta(z)=0$ is the only solution of Eq. (20) when $c(z)<c_{\mathrm{UB}}(z)$, i.e., below the local bifurcation density. This feature of the UB bifurcation is qualitatively different from the bulk IN bifurcation discussed earlier, where nematic solutions continue to exist at densities (not too far) below the bifurcation. The difference is caused by the different nature of the broken symmetry in the two cases, as discussed in detail by Mulder. ${ }^{34}$ It follows from the third of the EulerLagrange equations (8), with $V_{\alpha}(z)=V_{\alpha}^{(1)}(z)$, that $c_{3}(z)$ $=0$, and hence $s(z)=-\frac{1}{2}$, for $0<z<L / 2$. Given that $-\frac{1}{2}$ is the minimum value for $s(z)$, and that $c_{\mathrm{UB}}(z)$ decreases with decreasing $s(z)$, we conclude that local biaxiality $\Delta(z) \neq 0$ 
starts to develop if $c(z)=1$, or $c_{1}(z)=c_{2}(z)=\frac{1}{2}$, anywhere in the interval $0<z<L / 2$. Inserting this condition in the first of Eqs. (8) at $z=0$ we find that the corresponding chemical potential $\beta \mu_{\mathrm{UB}}=1-\ln 2 \simeq 0.31<\beta \mu_{\mathrm{IN}}$. Thus, the wallinduced UB bifurcation occurs at bulk density $c_{\mathrm{UB}}^{*}$, which from Eq. (13) (with $s_{b}=0$ since the bulk is isotropic) is given by

$$
\ln \frac{2 c_{\mathrm{UB}}^{*}}{3}+\frac{4 c_{\mathrm{UB}}^{*}}{3}-1=0 \Rightarrow c_{\mathrm{UB}}^{*}=1.0311168 \ldots
$$

It follows that $c_{\mathrm{UB}}^{*} / c_{I} \simeq 0.819$, which is similar to the corresponding ratio 0.847 for freely rotating, infinitely elongated spherocylinders, extracted from Poniewierski's analysis. ${ }^{17}$ Note that the present analysis of the UB bifurcation is identical in spirit to that of Ref. 17, but is technically much less demanding because of the relative simplicity of the Zwanzig model.

We now turn attention to numerically determined profiles $c(z), s(z)$, and $\Delta(z)$ for a fluid of bulk density $c_{b}$ $<c_{I}$ in contact with a hard wall. These calculations involve the iterative solution of Eqs. (11) with a boundary condition $c_{\alpha}(z)=c_{b} / 3$ for $z \rightarrow \infty$ and $\alpha=1,2,3$. The actual calculations were performed on a grid with at least 100 points per $L$ for $0 \leqslant z / L \leqslant 10$ or 20 , which proved sufficient in all cases. In Fig. 2(a) we show $c(z)$ for several bulk densities $c_{b}<c_{\mathrm{UB}}^{*}$ (dashed curves) and $c_{b}>c_{\mathrm{UB}}^{*}$ (full curves); in Fig. 2(b) the corresponding order parameter profiles $s(z)(<0)$ and $\Delta(z)$ $\times(\geqslant 0)$ are shown. The sharp features at $z=L / 2$ are caused by the discontinuity of $c_{3}(z)$, which forces $s(z)=-1 / 2$ for $z<L / 2$. For all $c_{b}<c_{\mathrm{UB}}^{*}$ we find $\Delta(z)=0$ identically, even if the initial profile of the iterative process is chosen to be biaxial. For $c_{b}>c_{\mathrm{UB}}^{*}$ the $z$ interval where $\Delta(z)$ is nonzero increases steadily for increasing $c_{b}$, signifying the film with in-plane nematic ordering has increasing thickness. The onset of biaxiality is seen to be sharp. Although the biaxial character develops spontaneously for any initial profile of the iterative process if $c_{b}>c_{\mathrm{UB}}^{*}$, it is also possible to enforce uniaxial symmetry in the iterative process in this density regime. By comparing the interfacial tensions of these enforced uniaxial profiles with those of the spontaneously formed biaxial profiles at the same value of $c_{b}$ (or $\mu$ ), we find that the latter are always smaller. In the limit $c_{b} \rightarrow c_{\mathrm{UB}}^{*+}$ the difference is vanishingly small, while the gradients of the tensions as a function of $c_{b}$ are identical (see also Fig. 6). Thus, within the numerical accuracy it is tempting to conclude that the UB transition is of second order. This conclusion is supported by expanding the logarithm in Eq. (20). For $0<z \leqslant L / 2$ we find in the limit $\left(c_{b}-c_{\mathrm{UB}}^{*}\right) \rightarrow 0^{+}$that $\Delta(z)$ $\simeq \sqrt{3\left(c(z)-c_{\mathrm{UB}}(z)\right)}$, with $s(z)=-1 / 2$ and $c_{\mathrm{UB}}(z)=1$. Assuming $c(z)$ remains smooth in the vicinity of the origin this indicates standard mean-field behavior of the order parameter at the transition. Our result differs from that of Ref. 18, where the UB transition of a system of semiflexible polymers near a hard wall is predicted to be very weakly first order. Whether this signals a fundamental difference between the two models or whether it reflects a limited numerical resolution is not clear at this stage. Note that beyond mean field we do expect to find differences between models with restricted orientations, such as the Zwanzig model, and those without
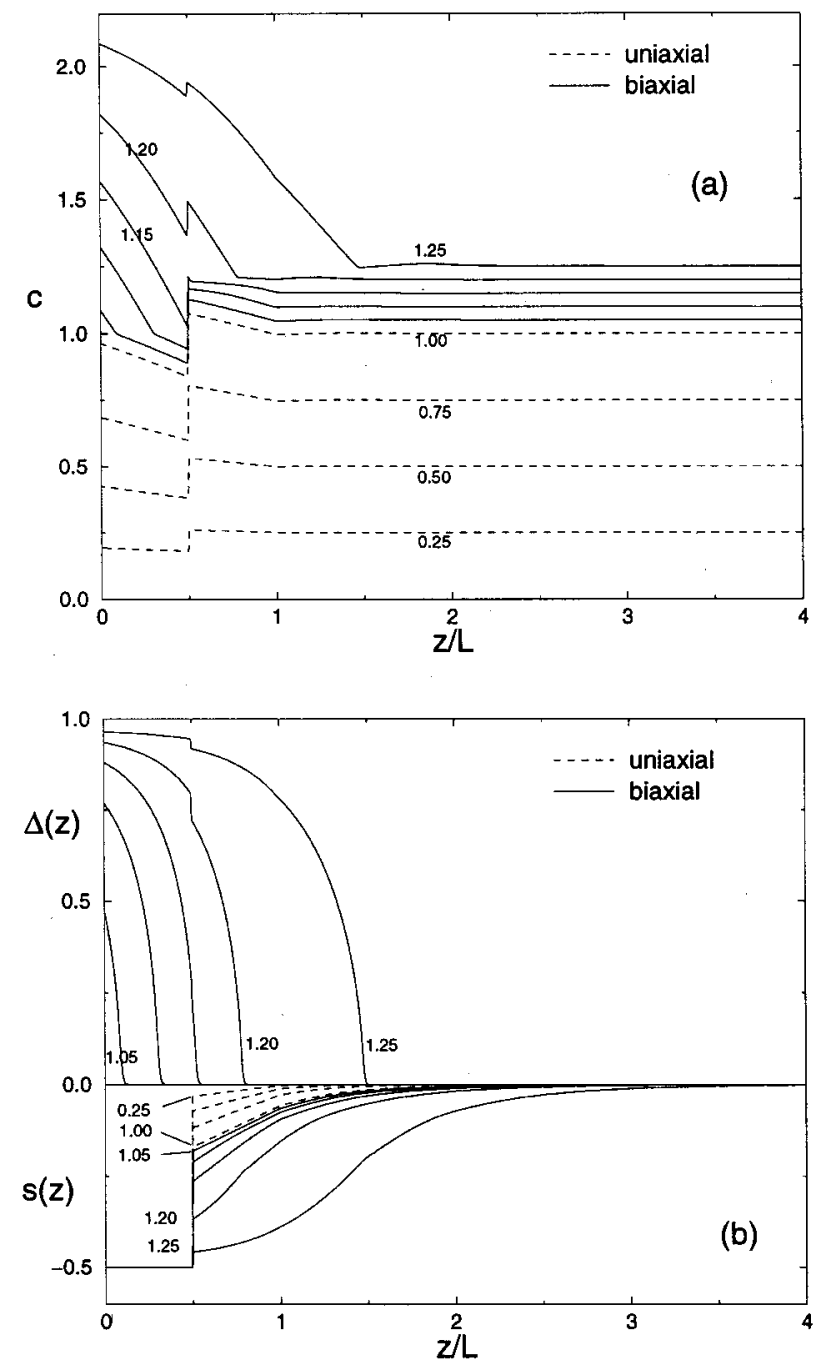

FIG. 2. Profiles of a hard-rod fluid in contact with a single hard wall located at $z=0$. The bulk density $c_{b}$ far from the wall takes values $c_{b}=0.25,0.50$, 0.75 , and 1.00 (dashed curves, uniaxial profiles) and 1.05, 1.10, 1.15, 1.20, and 1.25 (full curves, biaxial profiles). The profiles in (a) represent the total density $c(z)$, and in (b) the orientational order parameters $s(z)$ and $\Delta(z)$. Note that $\Delta(z) \equiv 0$ for uniaxial profiles, i.e., for $c_{b}<c_{\mathrm{UB}}^{*}=1.0311168$ and that $s(z)=-0.5,0 \leqslant z \leqslant L / 2$, for all values of $c_{b}$.

restrictions. Due to the absence of a soft mode, the in-plane correlations in the $B$ phase should be long ranged in the case of the Zwanzig model, whereas the soft mode that exists in systems with continuous orientations will, probably, destroy the long-range in-plane order. The UB transition has been discussed for thermotropic liquid crystals within the context of Landau-de Gennes theory; it can be first order or continuous. ${ }^{35,36}$ Ascertaining the precise nature of this transition in hard-rod models requires further investigation. It is also interesting to consider the similarities and differences between the present UB transition and the prewetting transition observed for simple fluids in contact with a sufficiently attractive wall. ${ }^{37}$ The two transitions are similar in the sense that the surface "triggers" a phase transition (here the onset of orientational ordering close to the wall; in a simple fluid a thin-thick transition of the liquid-like film close to the wall) at a lower chemical potential than that required for the corresponding bulk transition (here the IN transition; in a simple 


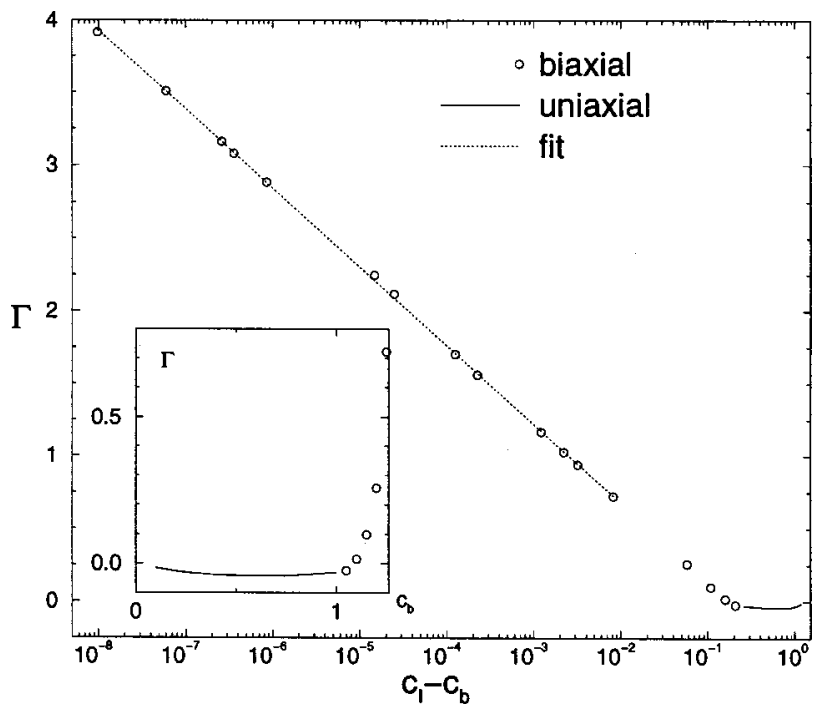

FIG. 3. Adsorption $\Gamma$ of a fluid of hard rods in contact with a single hard wall, as a function of the bulk density $c_{b}$ approaching (from below) the bulk isotropic-nematic coexistence density $c_{I}$. The straight line indicates a logarithmic divergence as $c_{b} \rightarrow c_{I}^{-}$. This is a signature of complete wetting of the wall-isotropic fluid interface by a nematic film. The inset shows the results on a linear $c_{b}$ scale, and displays a change of sign of $\Gamma$ for $c_{b}$ $\approx 1.10$. Solid lines denote uniaxial profiles and the symbols denote biaxial profiles. The dotted line is a fit to the data near $c_{l}$-see text.

fluid the gas-liquid transition). An important difference between the two transitions is that prewetting is first order (accompanied by a discontinuous jump of the film thickness from a small nonzero value to a larger finite value), while the UB transition is continuous; the biaxial film thickness develops from identically zero to nonzero values. This last point is crucial for understanding the insensitivity of the UB transition to confinement, as discussed later. Note also that prewetting is a precursor of the wetting transition that occurs at bulk coexistence, ${ }^{37}$ whereas the UB transition is not intimately related to any wetting transition.

It can be seen in Fig. 2(a) that the contact density $c(z$ $=0)$ is smaller than the bulk value $c(z \rightarrow \infty)=c_{b}$ at low $c_{b}$, but larger for $c_{b} \geqslant 1.1$. This signals a change in sign of the (dimensionless) adsorption $\Gamma$ as a function of $c_{b}$, where $\Gamma$ is defined as

$$
\Gamma=\frac{1}{L} \int_{0}^{\infty} d z\left(c(z)-c_{b}\right) .
$$

In Fig. 3 we plot $\Gamma$ as a function of $c_{b}<c_{I}$, i.e., in the regime where the bulk fluid in contact with the wall is isotropic. The full curve represents the uniaxial regime $c_{b}$ $\leqslant c_{\mathrm{UB}}^{*}$, and the symbols the biaxial regime. The inset of Fig. 3 , with the linear $c_{b}$ representation, displays the sign change at $c_{b} \simeq 1.1$. The main figure indicates that $\Gamma$ diverges logarithmically as $c_{b} \rightarrow c_{I}^{-}$. The straight line can be observed over several decades because of the accurate determination of $c_{I}$. It can be fitted by $\Gamma=A_{1}-A_{2} \ln \left(c_{I}-c_{b}\right)$, with fit parameters $A_{1} \simeq-0.404$ and $A_{2} \simeq 0.235$. The result of this fit is represented by the dotted line in Fig. 3. Combining the results of Figs. 2 and 3 suggests that a nematic film of increasing thickness (corresponding to an increasing adsorption $\Gamma$ ) develops as $c_{b} \rightarrow c_{I}^{-}$. The logarithmic increase of $\Gamma$ implies ${ }^{38}$

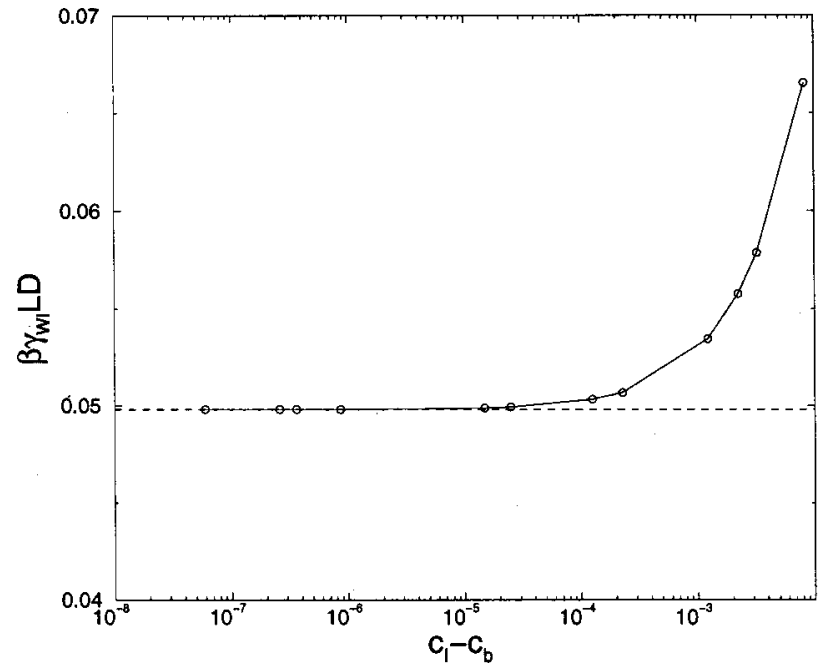

FIG. 4. The surface tension $\gamma_{\mathrm{WI}}$ of the hard wall-isotropic fluid interface as a function of the bulk density $c_{b}$ far from the wall, with $c_{b}$ in the regime slightly below the bulk isotropic-nematic transition density $c_{I}$. The limiting value (dashed line) is $\beta \gamma_{\mathrm{WI}}^{*} L D=(4.980 \pm 0.001) \times 10^{-2}$, which is the estimate of the tension at bulk coexistence.

that complete wetting of the wall-isotropic (WI) fluid interface by a nematic film should occur at $c_{b}=c_{I}$, where the nematic director of the film is oriented parallel to the wall. This complete wetting scenario is confirmed by a calculation of the contact angle $\vartheta$, defined by

$$
\cos \vartheta=\frac{\gamma_{\mathrm{WI}}^{*}-\gamma_{\mathrm{WN}, \|}^{*}}{\gamma_{\mathrm{IN}, \|}}
$$

where $\gamma_{\mathrm{WI}}^{*}=\gamma_{\mathrm{WI}}\left(c_{b}=c_{I}\right) \quad$ and $\quad \gamma_{\mathrm{WN}, \|}^{*}=\gamma_{\mathrm{WN}, \|}\left(c_{b}=c_{N} ; s_{b}\right.$ $=s_{N}$ ) denote the interfacial tension, defined in Eq. (17), between the wall-isotropic fluid phase (with $c_{b}=c_{I}$ ) and the wall-parallel nematic phase (with $c_{b}=c_{N}$ and $s_{b}=s_{N}$ ), respectively; $\gamma_{\mathrm{IN}, \|}$ is given in Eq. (18). It is straightforward to evaluate $\gamma_{\mathrm{WN}, \|}^{*}$ by solving numerically the Euler-Lagrange equations, with the appropriate boundary conditions, and inserting the profiles into Eq. (6) to obtain $\Omega_{0}$. The tension follows from Eq. (17) with $p=p_{\mathrm{IN}}$. The result is $\beta \gamma_{\mathrm{WN}, \|}^{*} L D=(2.1844 \pm 0.0004) \times 10^{-2}$. Due to the increasing thickness of the nematic film as $c_{b} \rightarrow c_{I}^{-}$, we determine $\gamma_{\mathrm{WI}}^{*}$ by extrapolating $\gamma_{\mathrm{WI}}\left(c_{b}\right)$ calculated for $c_{b}<c_{I}$ to $c_{I}$. The results for several values of $c_{b}$ are represented by the symbols in Fig. 4; the dotted line denotes the estimate of the limiting value, which is $\beta \gamma_{\mathrm{WI}}^{*} L D=(4.980 \pm 0.001) \times 10^{-2}$. Insertion of these results into Eq. (24) gives $\cos \vartheta=0.9999$ \pm 0.0004 . Within numerical accuracy, this result is consistent with a vanishing contact angle $\vartheta$, i.e., $\gamma_{\mathrm{WI}}^{*}=\gamma_{\mathrm{WN}, \|}^{*}$ $+\gamma_{\mathrm{IN}, \|}$, and, thus, with complete wetting of the WI interface by the nematic phase. It is also consistent with the profiles displayed in Fig. 5, which reveal the film increasing in thickness and exhibiting increasing bulk nematic character as $c_{b}$ $\rightarrow c_{I}^{-}$. The dashed lines represent the values of the order parameters of the coexisting nematic bulk phase. Note the extremely small deviations from the bulk coexistence values in the thickest film shown in Fig. 5. For clarity we present two (equivalent) sets of linear combinations of the profiles in Fig. 5, viz. $c(z), s(z)$, and $\Delta(z)$ in (a), and $c(z), s^{\prime}(z)$ 

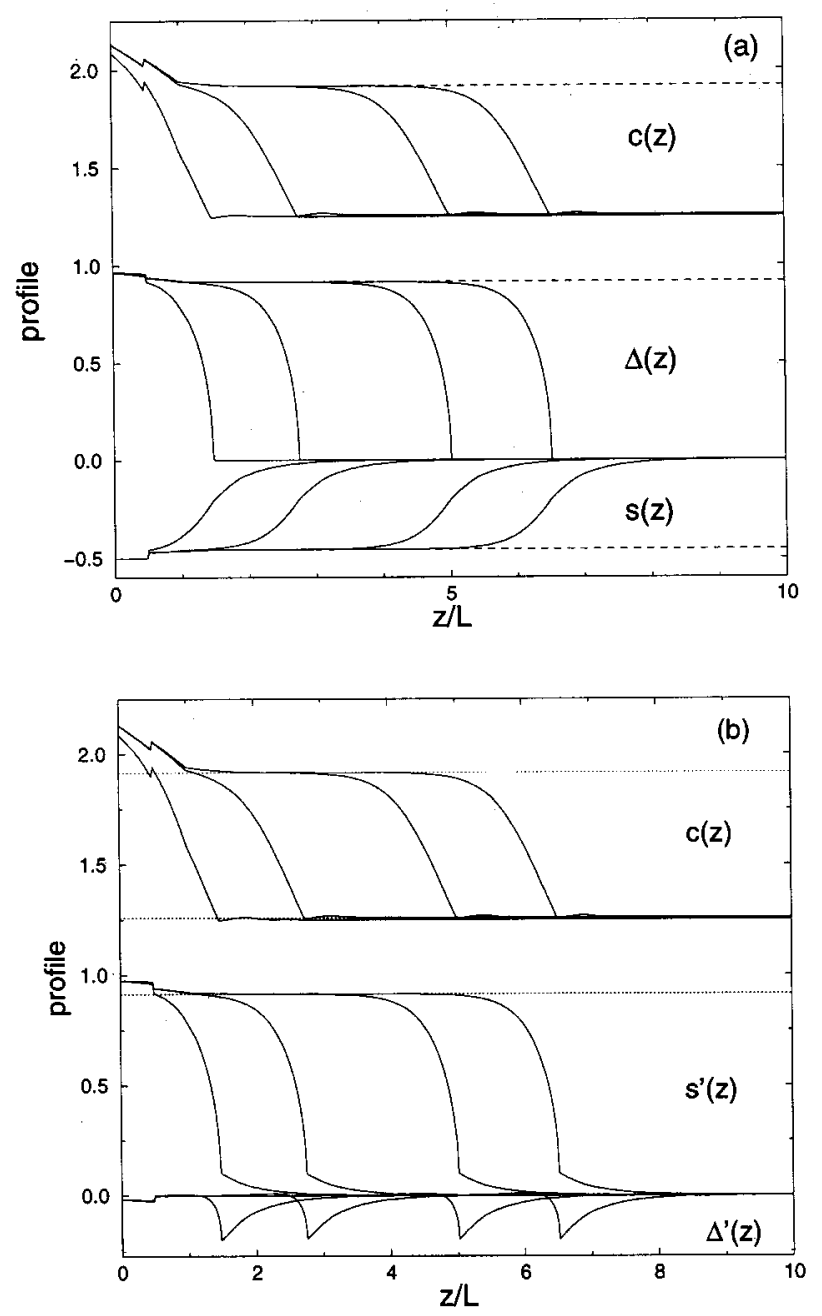

FIG. 5. Profiles of the interface between a single hard wall and an isotropic hard-rod fluid at a series of bulk densities $c_{b}=1.25,1.258,1.2582245$, and 1.25822485 , showing an intruding nematic film whose thickness diverges as $c_{b} \rightarrow c_{I}=1.25822486$. The curves in (a) and (b) are different representations (linear combinations) of the same profiles (see text). The dotted lines denote the values of the various order parameters in the coexisting bulk nematic phase. For the largest value of $c_{b}$ the interface between the nematic film and the isotropic bulk fluid [see (b)] mimics closely the free IN interface shown in Fig. 1.

$=-s(z) / 2+3 \Delta(z) / 4$, and $\Delta^{\prime}(z)=s(z)+\Delta(z) / 2$ in (b). The representation (a) shows directly the relation with the profiles in the lower $c_{b}$ regime of Fig. 2 [where $s(z)$ and $\Delta(z)$ are a "natural" choice], whereas the representation (b) allows for an easier comparison with the parallel (biaxial) bulk IN interface shown in Fig. 1. Clearly, the representations of the thick nematic films in Fig. 5(b) only show biaxial characteristics $\left(\Delta^{\prime}(z) \neq 0\right)$ close to the wall $(0<z<L / 2)$ and in the interface between the film and the isotropic bulk fluid. Comparison with all three profiles in Fig. 1 shows that the latter interface is virtually indistinguishable from the "free" IN interface between coexisting bulk phases, as expected for the case of complete wetting. As $c_{b} \rightarrow c_{I}^{-}$the interface depins from the wall. Note that the weak oscillation in the density $c(z)$ observed at the isotropic side of the IN interface persists for decreasing thickness of the nematic film.

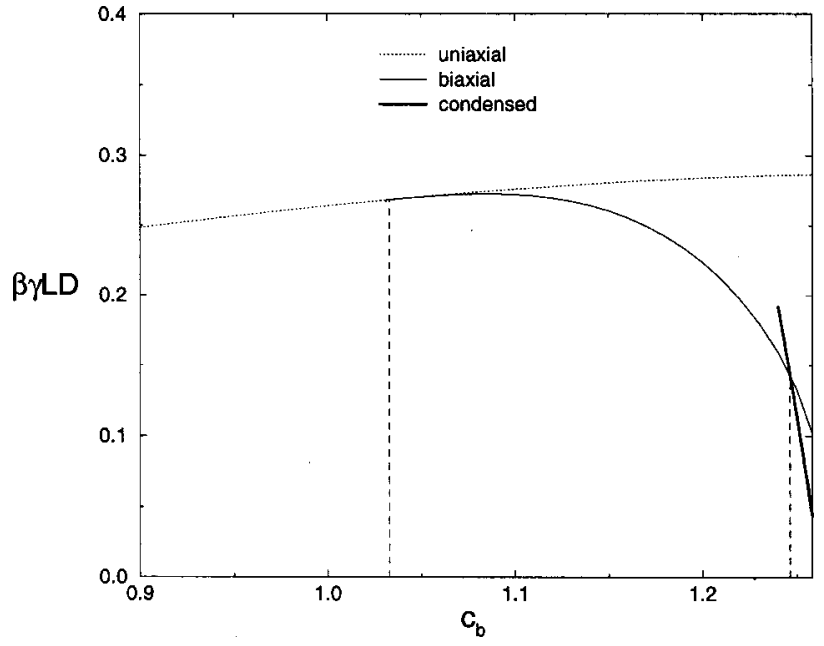

FIG. 6. Surface excess free energy $\gamma$ of the uniaxial, biaxial, and capillary condensed nematic phases of hard-rod fluid confined in a slit of relative width $h=H / L=6$ and in contact with an isotropic bulk reservoir at density $c_{b}$. The vertical dashed lines at $c_{b}=c_{\mathrm{UB}}=1.0313$ and $c_{b}=c_{\mathrm{BC}}=1.24643$ denote the location of the continuous uniaxial-biaxial and the first-order capillary nematization transition, respectively. Note that bulk coexistence occurs for $c_{b}=c_{I}=1.25822486$, which is the maximum $c_{b}$ displayed.

\section{THE FLUID CONFINED BY PARALLEL HARD WALLS}

We now consider the effect of confinement by two parallel hard walls on the phase behavior of the hard-rod fluid. Denoting the separation of these two walls by $H$, we characterize the walls by the external potential

$$
\beta V_{\alpha}^{(2)}(z)= \begin{cases}\infty & \text { for }|z|>H / 2 \quad(\alpha=1,2) \\ & 1|z|>(H-L) / 2 \quad(\alpha=3), \\ 0 & \text { otherwise }\end{cases}
$$

Our goal here is to determine the phase diagram of the system as a function of the relative slit width $h \equiv H / L$ and $\mu$, the chemical potential of the reservoir of rods. ${ }^{39} \mathrm{We}$ restrict our attention to $\mu \leqslant \mu_{\mathrm{IN}}$, and use the corresponding bulk density $c_{b} \leqslant c_{I}$ of the isotropic phase to characterize this chemical potential; the one-to-one correspondence between $\mu$ and $c_{b}$ is given in Eq. (13) with $s_{b}=0$.

The results presented here are based on the numerical solution of the Euler-Lagrange equations (8) with the external potential $V_{\alpha}(z)=V_{\alpha}^{(2)}(z)$ given in Eq. (25). In order to obtain the surface excess free energy $\gamma$, the equilibrium profiles are again inserted into Eq. (6) to obtain $\Omega_{0}$, and $\gamma$ follows from Eq. (17) with $p=p\left(c_{b}, s_{b}=0\right)$ from Eq. (13) and volume $V=A H$. In Fig. 6 we show the resulting surface excess free energy $\gamma$ as a function of $c_{b}$ for $h=6$. The dotted and thin full curves represent $\gamma_{U}$ and $\gamma_{B}$, the surface excess free energy for profiles with, respectively, uniaxial and biaxial symmetry close to the wall. These decay toward an (essentially) isotropic phase in the middle of the slit. For sufficiently low bulk densities, $c_{b}<c_{\mathrm{UB}} \simeq 1.0313$, the only profile satisfying the Euler-Lagrange equation is of uniaxial symmetry, and $\gamma_{B}$ is not defined. When $c_{b}>c_{\mathrm{UB}}$ a significant degree of biaxiality is possible close to the wall, and in this regime we find $\gamma_{B}\left(c_{b}\right)<\gamma_{U}\left(c_{b}\right)$, indicating that the biaxial phase is thermodynamically more favorable than the uniaxial 


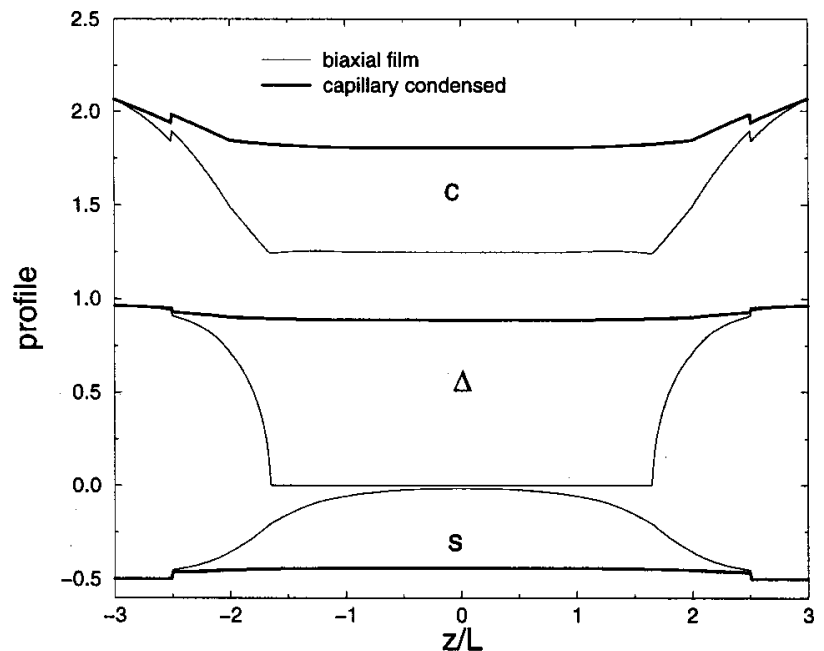

FIG. 7. Coexisting biaxial and capillary condensed nematic profiles for the hard-rod fluid in a slit of relative width $h=H / L=6$; the bulk (reservoir) density is $c_{b}=c_{\mathrm{BC}} \approx 1.246$. The two walls are at $z= \pm H / 2$. The biaxial profile is virtually isotropic in the central region, with total local density $c(z) \simeq c_{\mathrm{BC}}$, while the condensed phase bears strong resemblence to the coexisting nematic bulk phase with $c(z) \simeq c_{N}$ in the central region, and strong orientational ordering throughout the slit.

phase for $c_{b}>c_{\mathrm{UB}}$. The difference between $\gamma_{U}$ and $\gamma_{B}$ becomes vanishingly small as $c_{b} \rightarrow c_{\mathrm{UB}}^{+}$, and so does the difference between the gradients $\left(\partial \gamma / \partial c_{b}\right)$ of both curves. The reason for this is that the biaxiality $\Delta(z)$ vanishes in this limit and, hence, the difference between the uniaxial and biaxial profiles. Consequently, the UB transition at $c_{\mathrm{UB}}$ is continuous. This is completely equivalent to the one-wall situation considered in Sec. V. Our numerical estimate $c_{\mathrm{UB}}$ $\simeq 1.0313$ for $h=6$ is actually the smallest value of $c_{b}$ for which we distinguish between a uniaxial and biaxial profile, and is therefore an upperbound; it is larger than the analytically determined quantity $c_{\mathrm{UB}}^{*}$ for a single wall (equivalent to $h \rightarrow \infty$ ) by about $0.02 \%$. We regard this small difference between $c_{\mathrm{UB}}^{*}$ and $c_{\mathrm{UB}}$ at $h=6$ as numerically insignificant, i.e., the location of the UB transition has not been altered by confinement. For $c_{b}>1.23$ another type of profile emerges as a solution of the Euler-Lagrange equations. This profile is characterized by local biaxiality close to the wall, and a decay toward an (essentially) uniaxial nematic phase in the middle of the slit. We associate this profile with the existence of a capillary condensed nematic phase $(C)$ in the confining slit. The corresponding surface excess free energy $\gamma_{C}$ is represented by the thick curve in Fig. 6. At $c_{b}=c_{\mathrm{BC}} \simeq 1.24643$ we find that $\gamma_{C}$ intersects $\gamma_{B}$ with the difference in slope being nonzero. This signifies a first-order capillary nematization transition at $c_{b}=c_{\mathrm{BC}}$. In Fig. 7 we plot the coexisting biaxial and condensed profiles $c(z), s(z)$, and $\Delta(z)$ at this transition for $h=6$. For clarity we display the full interval $-h / 2 \leqslant z / L \leqslant h / 2$, although we exploit the mirror symmetry about the midplane $z=0$ in the actual calculations. It is clear from Fig. 7 that the profile of the $B$ phase has $\Delta(z) \neq 0$ close to the walls, but is virtually isotropic in the central region with total local density $c(z) \approx c_{\mathrm{BC}}$. In contrast, the capillary condensed nematic phase has a strong nematic character persisting throughout the central region, with a total local mid-

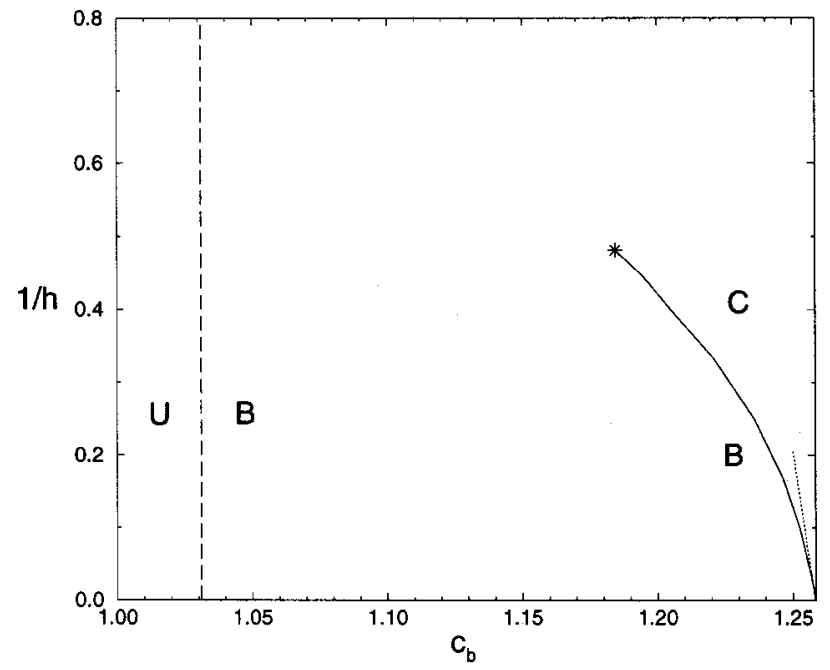

FIG. 8. Phase diagram of hard rods in a slit of relative width $h$ and in contact with a reservoir at bulk density $c_{b}$. The second order uniaxialbiaxial (UB) transition (dashed vertical line) takes place at $c_{b}=c_{\mathrm{UB}}^{*}$ $=1.031$, while the first-order biaxial-capillary nematization (BC) transition (full curve) shifts to lower $c_{b}$ with decreasing $h$ and ends in a critical point (*). The dotted line is the Kelvin approximation to the $\mathrm{BC}$ transition line, valid at large $h$ (see text).

plane density $c(0) \approx 1.79$ slightly below the coexisting nematic bulk density $c_{N}$. We note that the capillary condensed nematic phase $(C)$ is also biaxially symmetric close to the wall, but we omit this descriptor for convenience.

The UB and bulk coexistence (BC) transitions were determined for other values of the relative slit width $h$, and the phase diagram constructed as a function of $c_{b}$ and $1 / h$ is shown in Fig. 8. Here the UB and BC transitions are represented by the vertical dashed line and the full curve, respectively. For all $h>1$ the UB transition appears to be located at $c_{b} \simeq c_{\mathrm{UB}}^{*}$, the $h \rightarrow \infty$ result. Although there are nonsystematic deviations from the single wall value, these are probably insignificant being at most $0.4 \%$. The capillary nematization is more interesting, since $c_{B C}$ decreases significantly with decreasing $h$. Moreover, the first-order capillary nematization ends, as we shall argue in more detail below, in a capillary critical point at the critical slit width $h=h_{c} \simeq 2.08$ \pm 0.01 ; this critical point is denoted by the asterisk $(*)$ in Fig. 8. In the limit $h \rightarrow \infty$ we see that the BC phase boundary approaches the isotropic bulk coexistence value $c_{b}=c_{I}$ linearly in $1 / h$. This linear limiting behavior can be derived from the Kelvin equation, represented by the dotted line in Fig. 8. First we approximate the grand potential $\Omega_{B}$ of the biaxial phase at a given chemical potential $\mu$ close to $\mu_{\mathrm{IN}}$ (or bulk density $c_{b}$ close to $c_{I}$ ) by $\Omega_{B}=2 \gamma_{\mathrm{WI}}^{*} A-p(\mu) V$, and that of the condensed phase by $\Omega_{C}=2 \gamma_{\mathrm{WN}, \|}^{*} A-p^{+}(\mu) V$. The factors of two in the surface tension contributions stem from the fact that there are two walls now, and $p^{+}(\mu)$ is the pressure of the metastable nematic bulk phase arising for $\mu$ $<\mu_{\mathrm{IN}} \cdot{ }^{23}$ Using the results that $\cos \vartheta=1$ in Eq. (24), and that $\partial p^{(+)}(\mu) L^{2} D / \partial \mu=c_{b}^{(+)}(\mu)$, the density of the (metastable) bulk system, one obtains from the coexistence condition $\Omega_{B}=\Omega_{C}$ that the value of the chemical potential at BC coexistence, $\mu_{\mathrm{BC}}$, satisfies 


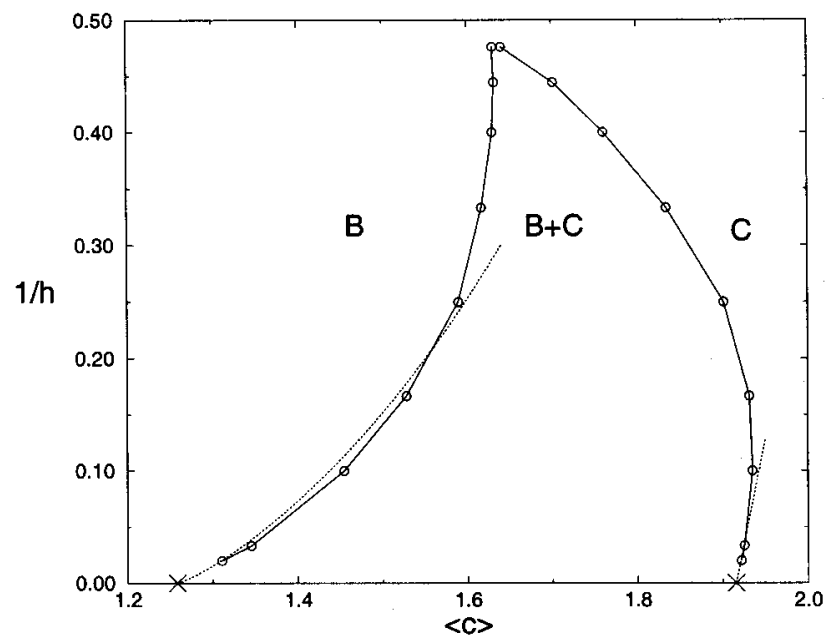

FIG. 9. The coexistence curve for the biaxial $(B)$ —capillary condensed nematic $(C)$ phase transition plotted as a function of average number density $\langle c\rangle$ in the slit and relative slit width $h$. The crosses $(\times)$ on the horizontal axis denote the bulk coexistence densities $c_{I}$ and $c_{N}$, and the dotted lines are large- $h$ approximations for the biaxial and capillary condensed phases (see text). The two branches of the coexistence curve intersect in the capillary critical point at $h=h_{c}=2.08$.

$$
\mu_{\mathrm{IN}}-\mu_{\mathrm{BC}}=\frac{2 \gamma_{\mathrm{IN}, \|} L D}{h\left(c_{N}-c_{I}\right)},
$$

which is the Kelvin equation appropriate to the present system. For sufficiently large $h$ the right hand side of Eq. (26) is arbitrary small but positive. Hence, one can expand the lefthand side about $c_{I}$, using $\left.\left(\partial \beta \mu / \partial c_{b}\right)\right|_{c_{I}}=1 / c_{I}+4 / 3$ $\approx 2.1281$ from Eqs. (13) and (14), to give $\beta \mu_{\mathrm{IN}}-\beta \mu_{\mathrm{BC}}$ $\approx 2.1281\left(c_{I}-c_{\mathrm{BC}}\right)$. Combining this result with Eq. (26), and using the numerical values of $c_{I}, c_{N}$, and $\gamma_{\mathrm{IN}, \|}$ yields the parameterisation $1 / h=A_{0}\left(c_{I}-c_{\mathrm{BC}}\right)$, with $A_{0} \simeq 25.0$, for the Kelvin line shown in Fig. 8. A detailed comparison of the prediction of the Kelvin equation with the full numerical results reveals a relative difference in $h^{-1}$ of order 0.05 for $c_{\mathrm{BC}}=1.2574$ (where $h=50$ ), and of order 0.4 for $c_{\mathrm{BC}}$ $=1.2525$ (where $h=10$ ); for smaller $c_{\mathrm{BC}}$ (or $h$ ) the Kelvin equation becomes increasingly poor. Similar tests of the Kelvin equation have been made for simple fluids in slit pores $^{23}$ and for a lattice model of the IN transition in finite films with free surfaces. ${ }^{40}$

In order to gain more insight into both the large and small- $h$ regime of capillary nematization we consider an alternative representation of the transition. This representation involves the average (dimensionless) number density $\langle c\rangle$ in the slit, defined as

$$
\langle c\rangle=\frac{1}{H} \int_{-H / 2}^{H / 2} d z c(z)=c_{b}+\frac{\Gamma}{h},
$$

where the adsorption $\Gamma$ is defined as in Eq. (23) but with the $z$-integration limits equal to $\pm H / 2$. Converting the $\mathrm{BC}$ phase boundary of Fig. 8 to the $\langle c\rangle-h^{-1}$ representation yields the two full curves in Fig. 9, where the low- $\langle c\rangle$ curve represents the coexisting $B$ phase and the high- $\langle c\rangle$ curve the corresponding $C$ phase. Obviously, the different values of $\langle c\rangle$ in the coexisting phases arise from different values of $\Gamma$, since $c_{b}$ and $h$ are identical. The two dotted curves represent parameterisations based on the following large- $h$ approximations. For the $B$ branch, we have $c_{b} \rightarrow c_{I}^{-}$and $\Gamma \rightarrow 2\left[A_{1}\right.$ $-A_{2} \ln \left(c_{I}-c_{b}\right)$ ], where the factor of 2 accounts for the confined case with two walls, and where $A_{1}$ and $A_{2}$ are the fit parameters for the single wall adsorption shown in Fig. 3. Combining this with the large- $h$ parameterization $h^{-1}$ $\simeq A_{0}\left(c_{I}-c_{b}\right)$ which follows from the Kelvin equation as discussed above, one obtains $\langle c\rangle \rightarrow c_{I}+2\left(A_{1}+A_{2} \ln \left(A_{0} h\right)\right) / h$ for the large- $h$ limit of the biaxial branch and this is shown in Fig. 9 as a dotted curve. For large $h$ the condensed nematic branch has the parameterization $\langle c\rangle \rightarrow c_{N}+2 \Gamma_{\mathrm{WN}, \|} / h$, with $\Gamma_{\mathrm{WN}, \|} \simeq 0.135$ the adsorption of the coexisting nematic bulk fluid at a hard wall in the parallel director geometry. We see that for small $h^{-1}$ the full curves of Fig. 9 are fairly well approximated by these asymptotic results, especially for the $C$ branch.

We focus now on the small- $h$ regime of Fig. 9, where the difference of average density between the coexisting $B$ and $C$ phase becomes progressively smaller and vanishes as $h \rightarrow h_{c}^{+}$and $c_{b} \rightarrow c_{\mathrm{BC}, c}$, with $h_{c}=2.08 \pm 0.01$ and $c_{\mathrm{BC}, c}$ $=1.184 \pm 0.001$. Such a vanishing density difference implies that the coexisting $B$ and $C$ phase coalesce in a critical point at a critical relative wall separation $h_{c}$ and a critical bulk density $c_{\mathrm{BC}, c}$. That this is a genuine critical point is confirmed by an analysis of the spatially integrated uniaxial and biaxial order parameters $S$ and $\Delta$, respectively, at $B C$ coexistence. $S$ and $\Delta$ are defined by

$$
S=\frac{1}{L} \int_{-H / 2}^{H / 2} d z s(z), \quad \Delta=\frac{1}{L} \int_{-H / 2}^{H / 2} d z \Delta(z) .
$$

Our results show that not only the values of $\Gamma$ (and hence $\langle c\rangle)$ become indistinguishable in the two phases as $h \rightarrow h_{c}^{+}$, but also those of $S$ and $\Delta$. This implies that the two phases are fully indistinguishable as $h \rightarrow h_{c}^{+}$and $c_{b} \rightarrow c_{\mathrm{BC}, c}$. The peculiar shape of the coexistence curve in Fig. 9, in particular the re-entrant features on both branches and the curvature near the critical point, should not be interpreted as indicating nonclassical behavior. The shape reflects the fact that the difference in $\langle c\rangle$ between the two phases was chosen as the order parameter. Other measures of the adsorption, for instance $\Gamma$ as a function of $\mu$ at fixed $h$, lead to more conventional shapes, with mean-field order parameter exponent $\beta=1 / 2$. We anticipate, as in the case of simple fluids, ${ }^{23}$ that in reality (beyond mean field) the capillary criticality of the Zwanzig model should lie in the two-dimensional Ising universality class since the order parameter is a scalar and correlations can only diverge in the $x-y$ plane.

For slit widths $h<h_{c}$ no capillary nematization takes place, and the slit "fills" continuously as $c_{b}$ is increased. This is illustrated in Fig. 10 for $h=2$, which is (slightly) below the critical slit width. In Fig. 10(a) the total density profile $c(z)$ is shown for various values of the bulk density $c_{b}$ in the range $1.05 \leqslant c_{b} \leqslant 1.25$. Figure 10 (b) displays the corresponding biaxiality profiles $\Delta(z)$. Note that the lowest value of $c_{b}>c_{\mathrm{UB}}^{*}$ so there is already a (thin) biaxial film present on each wall. This grows in thickness with increasing $c_{b}$. Although the character of the profiles appears to change significantly for $c_{b}$ in the range $1.17<c_{b}<1.18$, there is no 

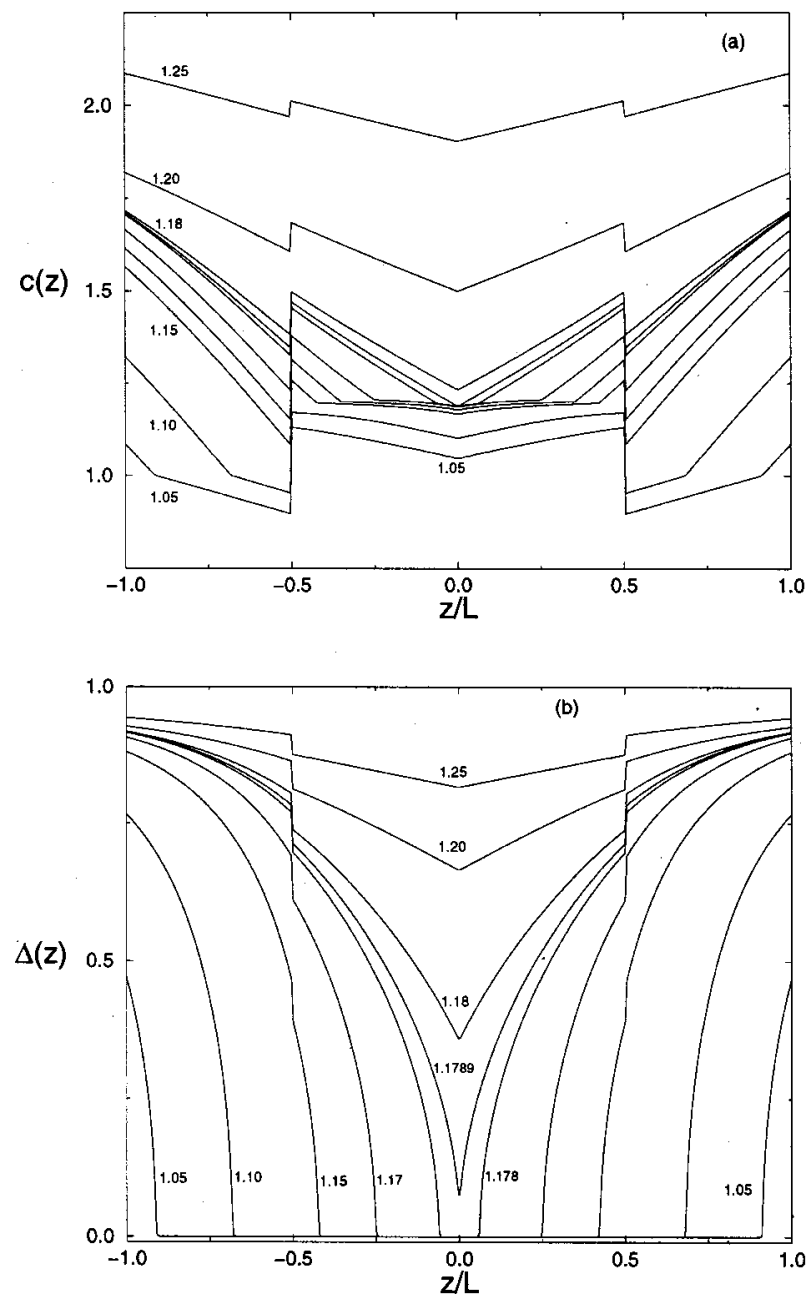

FIG. 10. Profiles of (a) total density $c(z)$ and (b) biaxial order parameter $\Delta(z)$ for confined hard rods, with relative slit width $h=2$, at the indicated values of bulk reservoir density $c_{b}$. Since $h<h_{c}$, no capillary condensation takes place; rather the capillary "fills" continuously as $c_{b}$ increases.

discontinuous change. For $c_{b}<c_{b}^{*} \simeq 1.1785$, we have $c(z$ $=0) \approx c_{b}$ and $\Delta(z=0)=0$. The two separate regions of surface biaxiality meet at $z=0$ when $c_{b}=c_{b}^{*}$, and for $c_{b} \geqslant c_{b}^{*}$ it is seen that $c(z=0)$ increases rapidly, eventually to be of the order of $c_{N}$ while $\Delta(z=0)$ grows from zero to large values characteristic of a nematic filling the slit. Consistent with the absence of a phase transition, we were unable to find two different profiles at the same $c_{b}$ for $h<h_{c}$.

We note that our calculated phase diagram is consistent with that obtained in Ref. 25, where the effect of confinement on a lattice model of a thermotropic liquid crystal was considered. These authors investigated the phase behavior as a function of the strength $\alpha$ of the surface field; our results correspond to a fixed, large value of $\alpha$ if one substitutes the coupling constant $J$ of their model for the chemical potential or $c_{b}$ of the present Zwanzig model.

\section{SUMMARY AND DISCUSSION}

We have studied certain aspects of surface phenomena in hard-rod fluids by considering the simple Zwanzig model in the limit of length-to-diameter ratio $L / D \rightarrow \infty$. The rectangu- lar shape of the rods and their restricted orientations permit, within the framework of the second virial free energy functional, a straightforward numerical evaluation of the density and order parameter profiles that minimize the functional. This is to be contrasted with equivalent treatments of (sphero)cylindrical or ellipsoidal rods with continuous orientation degrees of freedom, for which the determination of biaxially symmetric inhomogeneous profiles is numerically demanding. The present model suffers, of course, from a lack of realism compared with freely rotating hard rods, but we feel that this drawback is compensated, to some extent, by the accuracy with which the calculations can be performed and, therefore, with which phase behavior can be determined. Moreover, on the basis of comparisons with previous density functional results and with recent computer simulations of spherocylinders with continuous degrees of freedom, ${ }^{29,41}$ we argue that many predictions of the phase behavior emerging from the Zwanzig model actually hold for a wider class of hard-rod systems.

Having made a straightforward but accurate determination of the thermodynamic parameters specifying bulk IN coexistence, we study the "free" IN interface. We find that a nematic director parallel to the IN interface is thermodynamically more favorable than a director normal to the interface, consistent with earlier work on freely rotating hard rods. ${ }^{12-15}$ We also find significant biaxiality in the vicinity of the IN interface, similar to that found in Ref. 15 for spherocylinders. When the fluid of hard rods is in contact with a single planar hard wall, we find a continuous transition from uniaxial to biaxial symmetry near the wall at a density $c_{\mathrm{UB}}^{*}$ significantly below the IN bulk coexistence density $c_{I}$. Upon increasing the bulk density $c_{b}$ from $c_{\mathrm{UB}}^{*}$ toward $c_{I}$ our results show a continuously thickening nematic film intruding between the isotropic fluid and the wall, the nematic director of the film being parallel to the wall. The thickness of the film increases logarithmically with $\left(c_{I}-c_{b}\right)$, as is expected for short-ranged forces. Calculations of the three relevant surface tensions yield a contact angle that is zero, firmly establishing the complete wetting scenario, in agreement with previous findings for closely related models. ${ }^{16,18}$

In the final part of our study we consider a hard-rod fluid confined in a parallel slit of fixed width $H$. Apart from the continuous uniaxial-biaxial surface transition already seen in the one-wall system, we find a first-order capillary condensation (nematization) transition, provided the relative plate separation $h=H / L$ exceeds a critical value $h_{c} \simeq 2.08$. This first-order transition is found to terminate in a capillary critical point at $h=h_{c}$, and for $h<h_{c}$ there is a continuous "filling" of the slit as a function of $c_{b}$, the reservoir density. To the best of our knowledge, this is the first prediction of the existence of capillary coexistence and the accompanying capillary critical point in a hard-rod fluid. This prediction has been confirmed recently by Gibbs ensemble Monte Carlo simulations of freely rotating $L / D=15$ spherocylinders. ${ }^{29,41}$ These results illustrate how rich the entropically driven surface phase behavior is in such systems. 


\section{ACKNOWLEDGMENTS}

It is a pleasure to thank J.M. Romero-Enrique and L.F. Rull for helpful correspondence on the nomenclature of local nematic ordering near walls, M.P. Allen for providing a copy of Ref. 19 prior to publication, and P. van der Schoot for making us aware of Ref. 28. R.v.R. and M.D. thank Bristol University for their hospitality during the initial stages of this work, which was supported financially by Grant No. ERBFM-BICT971869 of the TMR program and Grant No. GR/L89013 of the EPSRC. This work is part of the research program of the Stichting voor Fundamenteel Onderzoek der Materie (FOM), which is supported financially by the Nederlandse Organisatie voor Wetenschappelijk Onderzoek (NWO).

${ }^{1}$ H. Zocher, Z. Anorg. Chem. 147, 91 (1925).

${ }^{2}$ F. C. Bawden, N. W. Pirie, J. D. Bernal, and I. Fankuchen, Nature (London) 138, 1051 (1936).

${ }^{3}$ L. Onsager, Ann. (N.Y.) Acad. Sci. 51, 627 (1949).

${ }^{4}$ For an excellent review see, e.g., G. J. Vroege and H. N. W. Lekkerkerker, Rep. Prog. Phys. 55, 1241 (1992).

${ }^{5}$ A. Stroobants, H. N. W. Lekkerkerker, and D. Frenkel, Phys. Rev. Lett. 57, 1452 (1986); Phys. Rev. A 36, 2929 (1987).

${ }^{6}$ A. M. Somoza and P. Tarazona, Phys. Rev. A 41, 965 (1990).

${ }^{7}$ A. Poniewierski and R. Holyst, Phys. Rev. A 41, 6871 (1990).

${ }^{8}$ M. Schmidt and H. Löwen, Phys. Rev. E 55, 7228 (1997).

${ }^{9}$ J. A. C. Veerman and D. Frenkel, Phys. Rev. A 41, 3237 (1990).

${ }^{10}$ P. Bolhuis and D. Frenkel, J. Chem. Phys. 106, 666 (1997).

${ }^{11}$ X. Wen, R. B. Meyer, and D. L. D. Caspar, Phys. Rev. Lett. 63, 2760 (1989).

${ }^{12}$ R. Holyst and A. Poniewierski, Phys. Rev. A 38, 1527 (1988).

${ }^{13}$ B. G. Moore and W. E. McMullen, Phys. Rev. A 42, 6042 (1990).

${ }^{14}$ Z. Y. Chen and J. Noolandi, Phys. Rev. A 45, 2389 (1992).

${ }^{15}$ Z. Y. Chen, Phys. Rev. E 47, 3765 (1993).

${ }^{16}$ A. Poniewierski and R. Holyst, Phys. Rev. A 38, 3721 (1988).

${ }^{17}$ A. Poniewierski, Phys. Rev. E 47, 3396 (1993).

${ }^{18}$ Z. Y. Chen and S. M. Cui, Phys. Rev. E 52, 3876 (1995).

${ }^{19}$ M. P. Allen, Mol. Phys. 96, 1391 (1999).

${ }^{20}$ M. P. Allen, J. Chem. Phys. 112, 5447 (2000).
${ }^{21}$ Y. Mao, P. Bladon, H. N. W. Lekkerkerker, and M. E. Cates, Mol. Phys. 92, 151 (1997).

${ }^{22}$ R. Evans, U. Marini Bettolo Marconi, and P. Tarazona, J. Chem. Phys. 84, 2376 (1986)

${ }^{23}$ R. Evans and U. Marini Bettolo Marconi, J. Chem. Phys. 86, 7138 (1987); R. Evans, J. Phys.: Condens. Matter 2, 8989 (1990) and references therein.

${ }^{24}$ P. Sheng, Phys. Rev. A 26, 1610 (1982); T. J. Sluckin and A. Poniewierski, in Fluid Interfacial Phenomena, edited by C. A. Croxton (Wiley, New York, 1986), Chap. 5.

${ }^{25}$ M. M. Telo da Gama and P. Tarazona, Phys. Rev. A 41, 1149 (1990).

${ }^{26}$ R. Zwanzig, J. Chem. Phys. 39, 1714 (1963).

${ }^{27}$ Y. Raton, R. van Roij, J. Cuesta, and B. Mulder, in New Approaches to Problems in Liquid State Theory, edited by C. Caccamo, J. P. Hansen, and G. Stell, NATO ASI series C 529 (Kluwer Academic, New York, 1999), p. 139.

${ }^{28}$ J. P. Straley, J. Chem. Phys. 57, 3694 (1972).

${ }^{29}$ R. van Roij, M. Dijkstra, and R. Evans, Europhys. Lett. 49, 350 (2000).

${ }^{30}$ R. Evans, Adv. Phys. 28, 143 (1979).

${ }^{31}$ R. van Roij, P. Bolhuis, B. Mulder, and D. Frenkel, Phys. Rev. E 52, R1277 (1995).

${ }^{32}$ B. M. Mulder, Phys. Rev. A 35, 3095 (1987).

${ }^{33}$ M. P. Allen, G. T. Evans, D. Frenkel, and B. M. Mulder, Adv. Chem. Phys. 86, 1 (1993).

${ }^{34}$ B. Mulder, Phys. Rev. A 39, 360 (1989).

${ }^{35}$ T. J. Sluckin and A. Poniewierski, Phys. Rev. Lett. 55, 2907 (1985).

${ }^{36}$ R. Seidin, R. M. Hornreich, and D. W. Allender, Phys. Rev. E 55, 4302 (1997)

${ }^{37}$ J. W. Cahn, J. Chem. Phys. 66, 3667 (1977); C. Ebner and W. F. Saam, Phys. Rev. Lett. 38, 1486 (1977); For a review of wetting and prewetting see S. Dietrich in Phase Transitions and Critical Phenomena, edited by C. Domb and J. L. Lebowitz (Academic, New York, 1988), Vol. 12, p. 1.

${ }^{38}$ For systems with short-ranged wall-fluid and fluid-fluid potentials, as is the case for the present model, a logarithmic divergence of $\Gamma$ is expected in the approach to complete wetting.

${ }^{39}$ Using $1 / h$ as a "thermodynamic" variable is not uncommon in studies of capillary condensation-see, e.g., R. Evans and U. M. B. Marconi, Phys. Rev. A 32, 3817 (1985); E. Bruno, U. M. B. Marconi, and R. Evans, Physica A 141, 187 (1987) who discuss the competition between condensation and prewetting.

${ }^{40}$ M. M. Telo da Gama, P. Tarazona, M. P. Allen, and R. Evans, Mol. Phys. 71, 801 (1990).

${ }^{41}$ M. Dijkstra, R. van Roij, and R. Evans (unpublished). 